\title{
Distinct Oncogenic Transcriptomes in Human Mammary Epithelial Cells Infected With Cytomegalovirus
}

\author{
Sandy Haidar Ahmad ${ }^{1 \dagger}$, Sébastien Pasquereau ${ }^{1 \dagger}$, Ranim El Baba ${ }^{1}$, Zeina Nehme ${ }^{1}$, \\ Clara Lewandowski ${ }^{1}$ and Georges Herbein ${ }^{1,2 *}$
}

1 Pathogens \& Inflammation/EPILAB Laboratory, EA4266, Université de Franche-Comté, Université Bourgogne Franche-Comté (UBFC), Besançon, France, ${ }^{2}$ Department of Virology, Centre Hospitalier Universitaire (CHU) Besançon, Besançon, France

OPEN ACCESS

Edited by: Wen-Wei Chang, Chung Shan Medical

University, Taiwan

Reviewed by:

Juliet Spencer,

Texas Woman's University,

United States

Yong Du,

Hospital for Special Surgery,

United States

*Correspondence:

Georges Herbein

georges.herbein@univ-fcomte.fr

${ }^{\dagger}$ These authors have contributed equally to this work

Specialty section:

This article was submitted to

Viral Immunology,

a section of the journal

Frontiers in Immunology

Received: 08 September 2021 Accepted: 24 November 2021 Published: 22 December 2021

Citation:

Haidar Ahmad S, Pasquereau S,

El Baba R, Nehme Z, Lewandowski C and Herbein G (2021) Distinct

Oncogenic Transcriptomes in Human Mammary Epithelial Cells Infected With Cytomegalovirus.

Front. Immunol. 12:772160. doi: 10.3389/fimmu.2021.772160
Human cytomegalovirus is being recognized as a potential oncovirus beside its oncomodulation role. We previously isolated two clinical isolates, HCMV-DB (KT959235) and HCMV-BL (MW980585), which in primary human mammary epithelial cells promoted oncogenic molecular pathways, established anchorage-independent growth in vitro, and produced tumorigenicity in mice models, therefore named high-risk oncogenic strains. In contrast, other clinical HCMV strains such as HCMV-FS, KM, and SC did not trigger such traits, therefore named low-risk oncogenic strains. In this study, we compared high-risk oncogenic HCMV-DB and BL strains (high-risk) with low-risk oncogenic strains HCMV-FS, KM, and SC (low-risk) additionally to the prototypic HCMVTB40/E, knowing that all strains infect HMECs in vitro. Numerous pro-oncogenic features including enhanced expression of oncogenes, cell survival, proliferation, and epithelialmesenchymal transition genes were observed with HCMV-BL. In vitro, mammosphere formation was observed only in high-risk strains. HCMV-TB40/E showed an intermediate transcriptome landscape with limited mammosphere formation. Since we observed that Ki67 gene expression allows us to discriminate between high and low-risk HCMV strains in vitro, we further tested its expression in vivo. Among HCMV-positive breast cancer biopsies, we only detected high expression of the Ki67 gene in basal tumors which may correspond to the presence of high-risk HCMV strains within tumors. Altogether, the transcriptome of HMECs infected with HCMV clinical isolates displays an "oncogenic gradient" where high-risk strains specifically induce a prooncogenic environment which might participate in breast cancer development.

Keywords: HCMV, cytomegalovirus, oncogenesis, breast cancer, high-risk, low-risk

\section{INTRODUCTION}

Breast cancer is the most common cancer diagnosed among women and recognized as one of the main causes of death in women (1). Etiologic factors of breast cancer are classified into genetic and environmental risk factors (2), and among these latter viruses could be involved $(3,4)$. Furthermore, the DNA and gene products of several viruses have been identified in breast cancer biopsies (5). Human cytomegalovirus (HCMV), a ubiquitous pathogen belonging to the Herpesviridae family, 
has been detected in $90 \%$ of early and metastatic breast cancers (6-8). Likewise, HCMV DNA or antigens have been found in other malignancies including brain (glioblastoma, medulloblastoma), colon, prostate, and liver (7, 9-13). HCMV causes asymptomatic to mild infection in immunocompetent host, but it can lead to serious complications in the immunocompromised host and cancer patients $(14,15)$.

Although the growth of laboratory HCMV strains is restricted to fibroblasts, the clinical HCMV isolates infect a broad range of cells including epithelial cells, endothelial cells, monocytes, macrophages, fibroblasts, stromal cells, hepatocytes, smooth muscle cells, and neural stem/progenitor cells (16-19). Despite its known onco- and immunomodulatory effects, HCMV can transform primary human mammary epithelial cells (HMECs) in vitro as previously reported for the HCMV-DB and HCMV-BL strains $(20,21)$ in addition to the human embryonal lung fibroblasts (22). Moreover, blood monocyte, tissue macrophages, CD34+, and neural stem/progenitor are identified as HCMV reservoirs harboring latent virus $(17,23-28)$.

The different oncogenic abilities, cellular and molecular characteristics allowed us to classify HCMV strains into high or low-risk oncogenic strains. The high-risk strains promoted oncogenic molecular pathways, induced the expression of stemness markers, transformed epithelial cells, induced the appearance of polyploid giant cancer cells (PGCCs) in culture, established anchorage-independent growth in vitro, and produced tumorigenicity in mice models $(20,21)$. In contrast, the low-risk strains did not display such oncogenic features (21).

Herein, we compared six clinical HCMV strains for their oncogenic potential in HMECs using soft agar assay and transcriptomic analysis. Therefore, we compared the transcriptome profile of HMECs infected with the high-risk HCMV-BL strain versus the low-risk HCMV-FS, KM and SC strains. The transcriptome of HMECs infected with HCMV-BL presents oncogenic traits, favors cell cycling, cell proliferation, and epithelial-to-mesenchymal transition (EMT). However, the transcriptome of the HCMV-FS, KM and SC low-risk strains did not show any of the previously mentioned characteristics. HMECs infected with HCMV-TB40/E displayed a transcriptome phenotype similar to that of the HCMV-FS lowrisk strain. In agreement with these distinct transcriptome profiles observed, we noticed the highest transformation and mammosphere formation in HMECs infected with the HCMVBL high-risk strain, but not with the HCMV-FS, HCMV-KM and HCMV-SC low-risk ones. In line with the transcriptome phenotype observed in acutely infected HMECs, we perceived the preferential detection of high-risk HCMV strains along with the high expression of $\mathrm{Ki} 67$ in breast cancer biopsies, especially in the basal-like ones.

\section{MATERIALS AND METHODS}

\section{Cell Cultures}

HMECs were purchased from Life Technologies (Carlsbad, CA, USA). MDA-MB231 and MCF7 cells were provided by Institut Hiscia (Arlesheim, Switzerland). Human embryonic lung fibroblasts (MRC5) were purchased from RD-Biotech (Besançon, France). HMECs were cultivated in HMEC medium (Life Technologies) supplemented with HMEC supplement and bovine pituitary extract (Life Technologies). MDA-MB231, MCF7, and MRC5 were maintained in Dulbecco's modified Eagle medium (PAN- Biotech) supplemented with fetal bovine serum (Dutscher) and penicillin- streptomycin (Life Technologies). Cultures were free of mycoplasma (VenorGem classic mycoplasma detection, Minerva biolabs).

\section{HMECs Infection With HCMV}

HMECs were infected with HCMV-DB (KT959235), HCMV-BL (MW980585), HCMV-TB40/E (KF297339), HCMV-FS, HCMV-KM, and HCMV-SC. The HCMV clinical isolates were previously isolated in our laboratory $(20,21)$, except for HCMVTB40/E (29). Cell-free virus stocks of HCMV were grown in HMECs as described previously (21). Virus titers were determined through the quantification of HCMV load by realtime qPCR detection using KAPA SYBR FAST Master Mix, Omega Bio-TEK) and IE1 gene primers (sense, 5'-CGACGT TCCTGCAGACTATG-3'; anti-sense, 5'-TCCTCGGTCA CTTGTTCAAA-3') according to the manufacturer's protocol. Results were collected and analyzed using MxPro qPCR software.

\section{Soft Agar Colony Formation Assay}

Colony formation in soft agar seeded with HMECs uninfected, infected with the six strains listed above, MCF7 cells and MDAMB231 cells were assayed using Cell Biolabs Cytosolic Cell Transformation Assay kit (Colorimetric assay, CB135; Cell Biolabs Inc., San Diego, CA) as per the manufacturer's protocol. The detection and quantification of colony formation was assessed by MTT assay (Cayman Chemical, Ann Arbor, MI). Colonies were observed under an Olympus microscope (Olympus Corporation, Tokyo, Japan)

\section{Confocal Microscopy and Immunohistochemistry}

Confocal microscopy was performed as described previously (21). Cells were washed using 1X PBS then fixed and permeabilized using BD Cytofix/Cytoperm, and finally stained with anti-IE1 (pp72) antibody (ab53495, Abcam). To visualize the nucleus, DAPI (4', 6'-diamidino-2- phenylindole) staining was performed according to the manufacturer's protocol. Poststaining, confocal images were taken using $63 \mathrm{X}$ oil immersion objective lens with a LSM800 Carl-Zeiss confocal microscope 95 (Germany). The Ki67 immunohistochemistry (IHC) evaluation on breast biopsies was assessed using the monoclonal antibody MIB1 (Invitrogen).

\section{Flow Cytometry Analysis}

Uninfected HMECs and HCMV-infected HMECs were fixed and permeabilized for 20 minutes at $4^{\circ} \mathrm{C}$ using $100 \mu$ l Cytofix/ Cytoperm solution (BD Biosciences), washed twice with $1 \mathrm{X}$ PBS supplemented with $3 \%$ FBS and $0.1 \%$ Triton-X, and then re-suspended in 100ul of staining buffer (1X PBS, 3\% FBS, and 
$0.1 \%$ Triton-X). Cell staining was done using the anti-IE1 (CMV-pp72) antibody conjugated to PE (sc-69834, Santa Cruz Biotechnology). HMECs were incubated in the dark for 60 minutes at $4^{\circ} \mathrm{C}$. Cells were washed twice with $1 \mathrm{X}$ PBS supplemented with $3 \%$ FBS and $0.1 \%$ Triton-X and subjected to cytofluorometric analysis on a BD LSRFortessa X-20 (BD Biosciences) flow cytometer. Data obtained was analyzed and processed using FACSDiva software (BD Biosciences).

\section{Phylogenetic Analysis}

Phylogenetic analysis was performed on several HCMV strains [BL (accession number: MW980585); DB (accession number: KT959235); TB40/E (accession number: KF297339); and Davis (accession number: JX512198.1)]. Multi-sequence alignments (MSA) were implemented using CLUSTAL W. Phylogenetic tree was constructed by means of the neighbor-joining method. The conducted analysis was done using MEGA7 software (http:// www.megasoftware.net/).

\section{$\mathbf{R T}^{2}$ Profiler $^{\mathrm{TM}}$ PCR Arrays Experiment}

Total RNA was extracted from uninfected HMECs and HMECs infected with HCMV-BL, HCMV-TB40/E, HCMV-FS, HCMV$\mathrm{KM}$ and HCMV-SC at MOI of 1 at day 1 post-infection using an RNA extraction kit (EZNA Total kit I, Omega BIO-TEK). Then, the cDNA was synthesized using the $\mathrm{RT}^{2}$ First Strand Kit (Qiagen, Germantown, MD, USA) following the manufacturer's instructions. Two $\mathrm{RT}^{2}$ Profiler ${ }^{\mathrm{TM}}$ PCR Arrays for human breast cancer (PAHS-131ZA) and human oncogenes \& tumor suppressor genes (PAHS-502ZR) (both from Qiagen, Germantown, MD, USA) were performed using Mx3005P real-time PCR system as per manufacturer's instructions. These microarrays allow the quantification of mRNA from 84 cellular genes, using qPCR with premixed primer sets and SybrGreen qPCR MasterMix. DNA contamination control, housekeeping genes, reverse transcription control, and positive control were included in each PCR array. Data were analyzed using the manufacturer web-based analysis software (http://pcrdataanalysis.sabiosciencescom/pcr/arrayanalysis.php). The analysis was done based on three independent biological experiments for each of the HCMV strains.

\section{Mammosphere Assay}

Mammosphere formation by uninfected HMECs and HMECs infected with HCMV-BL, HCMV-TB40/E, HCMV-FS, and $\mathrm{HCMV}-\mathrm{KM}$ at MOI of 1 was assayed as described previously (19). MCF7 and MDA-MB231 were used as positive controls. Mammospheres larger than 60 microns were counted.

\section{Western Blotting}

Cellular extracts of uninfected and infected HMECs with HCMV-DB and HCMV-TB40/E were prepared at 1 hour, 5 hours, days 1 and 3 post-infection and used to assess the expression of Rb, pRb, Akt, pAkt-Th308, pAkt-Ser473, STAT3, pSTAT3, CyclinD1, and ATM as described previously (20). Protein levels were quantified using ImageJ 1.40 software (National Institutes of Health, Bethesda, MA, USA). $\beta$-actin was used as loading control to normalize sample loading.
Anti-Rb, anti-pRb, anti-Akt, anti-pAktThr308, anti-pAktSer473, anti-cyclin D1, and anti-ATM antibodies were purchased from Cell signaling (Danvers, MA, USA). Anti-pSTAT3 and anti-STAT3 antibodies were purchased from Santa Cruz Biotechnology (Santa Cruz, CA). Anti- $\beta$-actin antibody was purchased from SigmaAldrich (St. Louis, MO, USA).

\section{Breast Biopsies}

Nineteen frozen tumor breast biopsies (ten luminal tumor biopsies and nine basal tumor biopsies) and eight adjacent healthy breast biopsies were provided by the regional tumor bank (BB0033-00024 Tumorothèque Régionale de FrancheComté). Healthy breast biopsies were numbered from one to eight, luminal tumor biopsies from nine to eighteen, and basal tumor biopsies from nineteen to twenty-seven. A detailed histopathological biopsy data including the histologic biopsy type, Elston-Ellis grading system, hormone percentages (estrogen and progesterone), presence or absence of the human epidermal growth factor receptor 2 (HER2) protein, vascular emboli, and TNM staging was provided as a supplementary data (Supplementary Table 1). Additionally, using twenty different breast tumor biopsies, we assessed the Ki67 mRNA and Ki67 protein levels measured by RT-qPCR and IHC, respectively, on the same biopsy sample. A written informed consent for participation was obtained from all patients. The study was authorized by the local ethics committees of Besançon University Hospital (Besançon, France) and the French Research Ministry (AC-2015-2496, CNIL n¹173545, NF-S-138 $\left.96900 \mathrm{n}^{\circ} \mathrm{F} 2015\right)$. The presence of HCMV, within all the biopsies, was assessed by qPCR using IE1 and UL69 primers as mentioned above and described previously (30). For Ki67 and GAPDH mRNA quantification, RNA was extracted from the biopsies using E.Z.N.A. ${ }^{\circledR}$ Total RNA Kit I (Omega BIO-TEK, GA, USA). Reverse transcription was performed using the SuperScript IV First-Strand Synthesis kit (Invitrogen, Carlsbad, CA, USA). The expression of Ki67 and GAPDH was assessed by real-time qPCR using KAPA SYBR FAST Master mix (KAPA BIOSYSTEMS, KK4601) and specific primer for Ki67 (sense, 5'TCCTTTGGTGGGCACCTAA GACCTG3'; anti-sense, 5'TGATGGTTGAGGTCGTTCCTTG ATG3') and GAPDH (sense, 5'CCCCTCTTCAAGGCCTC TAC3'; anti-sense, 5'CGACCACTTTGTCAAGCTCA3') according to the manufacturer's protocol. The fold change expression was calculated using the delta-delta $\mathrm{Ct}$ method (31). Biopsies having a fold change for Ki67 gene expression $<30$, from 30 to 100 , and $>100$ were considered to have a low, intermediate, and high Ki67 mRNA absolute level respectively. The Ki67 mRNA cut-off was set based on the state of healthy biopsies (32).

\section{Statistical Analysis}

Statistical analysis was performed using the Wilcoxon test with the help of the Mathematics Department of the University of FrancheComté. p-value $\leq 0.05$ were considered statistically significant. In data analysis, Microsoft Excel was used to prepare the plots with 
standard deviation and p-values. Statistical significance was represented on figures by stars, with the following code ${ }^{*} p$ value $<0.05 ;{ }^{* *} p$-value $<0.01 ;{ }^{* *} p$-value $<0.001$.

\section{RESULTS}

\section{Growth and Transforming Capacity of Six HCMV Clinical Strains in HMECs}

A phylogenetic analysis was performed to analyse the genomic sequences of four HCMV strains, namely HCMV-DB, HCMVBL, HCMV-TB40/E, and HCMV-Davis. Since the clinical isolates FS, KM and SC genomes were not sequenced, we selected the strain Davis for its similar clinical characteristics (33). The phylogenetic analysis showed that HCMV-BL is close to HCMV-DB, and HCMV-TB40/E is close to the genomes of HCMV-Davis (Supplementary Figure 1). The two observable brackets illustrate a high-risk group including DB and BL strains, and a low-risk group including TB40/E and Davis strains (Supplementary Figure 1).

We assessed the replication of six HCMV clinical isolates in HMECs, namely the HCMV-BL and HCMV-DB high-risk strains, the HCMV-FS, HCMV-KM and HCMV-SC low-risk strains, and the prototypic TB40/E strain $(20,21,29)$. We evaluated the expression of the immediate early protein IE1 by immunofluorescence and flow cytometry. We observed strong IE1 expression in HMECs infected with the five strains (HCMVDB, BL, TB40/E, FS and KM) at day 1 post-infection compared to the uninfected HMECs by confocal microscopy (Figure 1A). Moreover, the expression of IE1 protein in HMECs infected with the different HCMV strains was confirmed by flow cytometric analysis (Figure 1B). Further, we noticed productive replication of all six HCMV strains in infected HMECs as assayed by IE1 detection in culture supernatants using qPCR (Figure 1C and Supplementary Figure 2A). An early and transient peak of viral replication was observed at day 1 post-infection for HMECs infected with HCMV-DB, HCMV-BL, and HCMV-TB40/E unlike the delayed peaks at day 3 and 5 post-infection for HMECs infected with HCMV-KM and HCMV-FS respectively. To further assess the oncogenic status of all six HCMV strains, we performed a soft agar assay. HMECs infected with the six HCMV strains were seeded in soft agar at day 1 post-infection. Cancer cell lines, MCF7 and MDA-MB231, were used as positive controls. Uninfected HMECs were used as negative control. Colony formation was detected at day 15 post-infection only with HCMV-DB and HCMV-BL compared to the uninfected HMECs ( $p$-value ${ }_{(\mathrm{HCMV}-\mathrm{DB}: \mathrm{UI} \text { HMEC) }}=0.03, p$-value ${ }_{(\mathrm{HCMV}-\mathrm{BL}: \mathrm{UI}}$ HMEC) $=0.03, p$-value $($ HCMV-DB : HCMV-TB40/E) $=0.03 ; p$-value $($ HCMV-DB $:$ HCMV-FS $)=0.03 ; p$-value $($ HCMV-DB $:$ HCMV-KM) $=0.03$; $p$-value ${ }_{\text {HCMV-BL : HCMV-TB40/E) }}=0.03 ; p$-value ${ }_{\text {HCMV-BL : HCMV- }}$ $\mathrm{FS})=0.03 ; p$-value (HCMV-BL : HCMV-KM) $=0.03$ ). In contrast, no significant colony formation was observed with HCMV-FS, HCMV-KM, HCMV-SC, and HCMV-TB40/E infected HMECs (Figure 1D and Supplementary Figure 2B). Our results indicate that HMECs infected with the high-risk strains BL and DB have an anchorage-independent growth ability.

\section{Distinct Transcriptome Profiles in HMECs Infected With Low-Risk and High-Risk HCMV Strains}

We decided to compare the transcriptome profile of the high-risk BL strain, the low-risk FS, KM and SC strains, and the prototypic TB40/E strain. Human breast cancer along with human oncogenes and tumor suppressor genes $\mathrm{RT}^{2}$ profiler PCR arrays were used to screen the infected HMECs with the four strains at MOI of 1 at day 1 post-infection and the uninfected HMECs. The expression of genes that are involved in signal transduction, cell cycle, cell survival, adhesion, angiogenesis, DNA repair, were examined by the $\mathrm{RT}^{2}$ Profiler PCR arrays (Supplementary Tables 2, 3).

The individual gene expression of the oncogenes $M y c$ (MYC), Fos (FOS), Jun (JUN), KRas (KRAS), and E2F1 was assessed in HMECs infected with the five strains HCMV-BL, TB40/E, FS, $\mathrm{KM}$ and SC compared to uninfected HMECs (Figures 2A, 3A and Supplementary Figure 2C). Values for individual genes were pooled to constitute the overall expression of the oncogenes group (Figure 2A). In HMECs infected with HCMV-BL, the expression of the oncogenes group was 5.8-fold higher compared to HMECs infected with the TB40/E strain ( $p$-value (HCMV-BL: HCMV-TB40/E) $=0.02), 8.5$-fold higher compared to HMECs infected with FS strain ( $p$-value (HCMV-BL: HCMV-FS) $=0.004$ ) (Figure 2A). However, the expression of these oncogenes was downregulated in HMECs infected with the KM strain ( $p$-value $($ HCMV-BL: HCMV-KM) $=0.0001)$ (Figure 2A). Among the oncogenes, Myc and E2F1 were mostly increased in HMECs infected with HCMV-BL compared to HCMV-TB40/E ( $p$-value (Myc- HCMV-BL: HCMV-TB40/E) $=0.12 ; p$-value $(\mathrm{E} 2 \mathrm{~F} 1-\mathrm{HCMV}-\mathrm{BL}$ : HCMV$\mathrm{TB} 40 / \mathrm{E})=0.04)$, HCMV-FS ( $p$-value $($ Myc- HCMV-BL: HCMV-FS) $=$ 0.12 ; p-value (E2F1- HCMV-BL: HCMV-FS) $=0.27$ ) and HCMV-KM

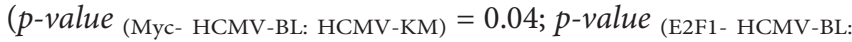
HCMV-KM) $=0.12$ ) infected HMECs (Figure 3A). Furthermore, the expression of other oncogenes was tested for HMECs infected with the high-risk strain BL and the low-risk strain FS versus uninfected HMECs. We observed an upregulation of the transcripts of numerous other oncogenes such as KITLG, MYB, RARA, ROS1, and RUNX1 in HMECs infected with HCMV-BL and HCMV-FS (Table 1). The upregulation of KITLG, RARA, and ROS1 genes expression was at least 2-fold higher in HMECs infected with HCMV-BL compared to HMECs infected with HCMV-FS (Table 1). In HMECs infected with HCMV-SC, the oncogenes' expression (MYC, FOS, KRAS, JUN, E2F1) was low, likewise in HMECs infected with HCMV-FS (Supplementary Figure 2C).

The individual gene expression of the tumor suppressor genes coding for $\mathrm{p} 53$ protein (TP53), retinoblastoma protein (Rb), p73 protein (TP73), SMAD4, VHL, TSC1, and MDM2 was also assessed in the previously mentioned five strains compared to uninfected HMECs (Figures 2B, 3B and Supplementary Figure 2C). Values for individual genes were pooled to constitute the overall expression of the tumor suppressor genes group (Figure 2B). The upregulation of tumor suppressor genes' expression was higher in HMECs infected with the BL strain compared to HMECs infected with the TB40/E and FS by 2 

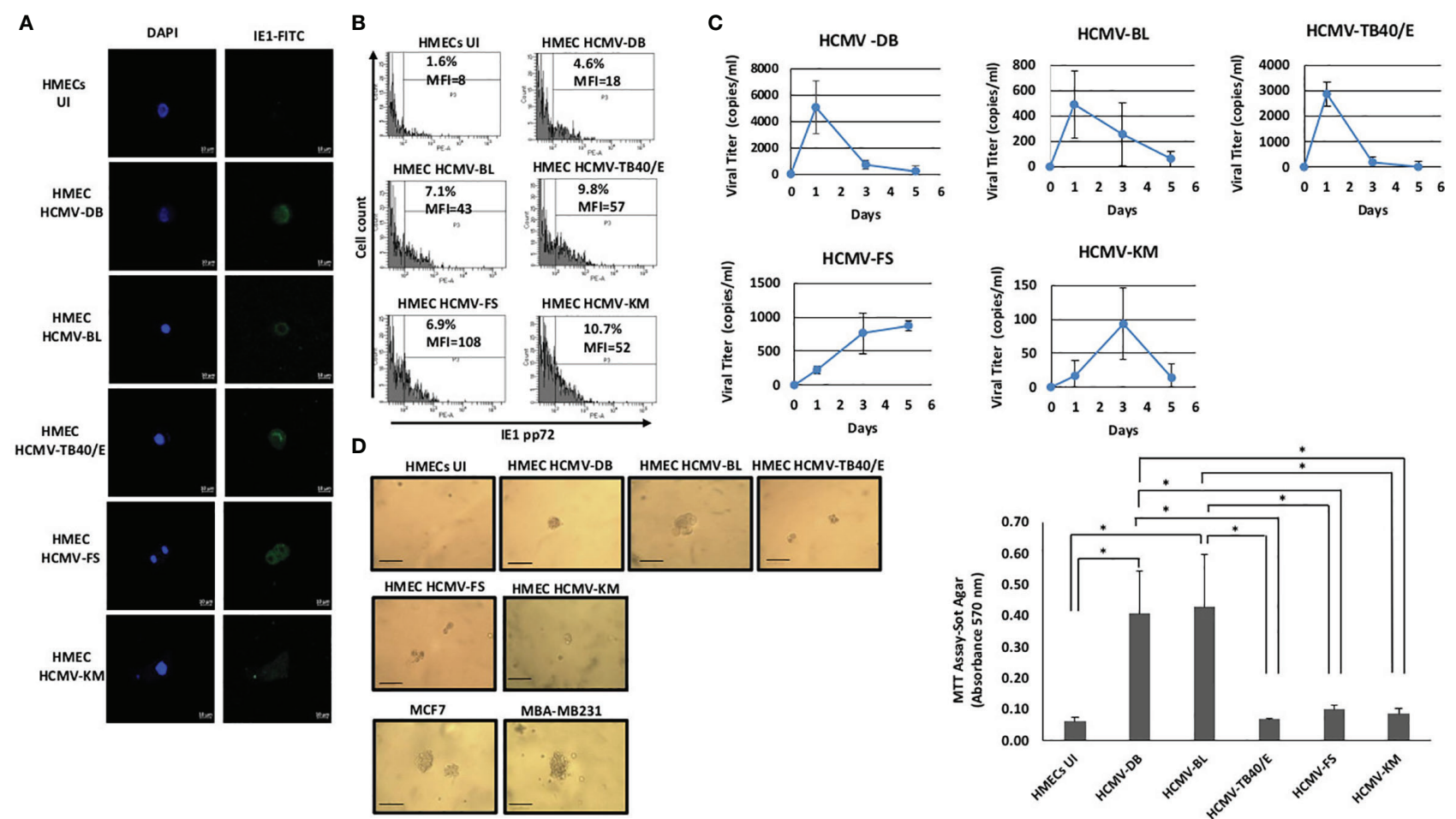

HCMV-KM
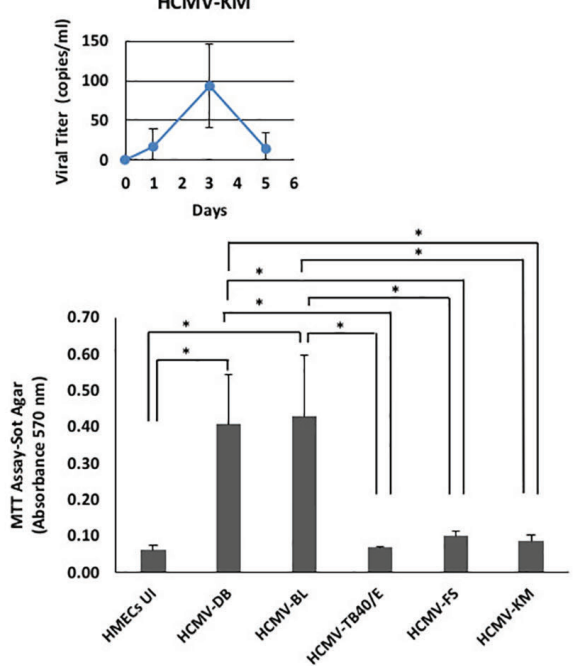

FIGURE 1 | Replication and transforming capacities of five HCMV strains in HMEC cultures. (A) Confocal microscopic images of DAPI and IE1 staining in HMECs infected with HCMV-DB, HCMV-BL, HCMV-TB40/E, HCMV-FS, and HCMV-KM. Uninfected HMECs (UI) was used as negative control. Magnification X63, scale bar $10 \mu \mathrm{m}$. Data shown are representative of three independent experiments. (B) Detection of IE1 (pp72) protein in HMECs infected with HCMV-DB, HCMV-BL, HCMV-TB40/E, HCMV-FS, and HCMV-KM. Uninfected HMECs (UI) was used as negative control. Data are represented as mean \pm SD of two independent experiments. (C) Growth kinetics of the five HCMV strains in HMECs up to day 5 post-infection. Measurement of IE1 by qPCR in order to assess the viral growth. Data are represented as mean \pm SD of three independent experiments. (D) Colony formation in soft agar seeded with HMECs infected with HCMV-DB, HCMV-BL, HCMV-TB40/E, HCMV-FS, and HCMV-KM at MOI of 1. MDA-MB231 and MCF7 were used as positive controls. Uninfected HMECs were used as negative control. Colonies were observed under an inverted light microscope (magnification $\times 100$, scale bar 100 um). Right panel. Quantification was performed at day 15 post-seeding. Histogram represents mean \pm SD of three independent experiments. $p$-values were determined by Wilcoxon test. ${ }^{*} p$-value $<0.05$.

$(p$-value $($ HCMV-BL: HCMV-TB40/E) $=0.02)$ and 3.8 fold $(p$-value $($ HCMV-BL: HCMV-FS $)=0.002)$ respectively (Figures $2 \mathbf{B})$. Nevertheless, the expression of these tumor suppressor genes was downregulated in HMECs infected with HCMV-KM ( $p$ value $(\mathrm{HCMV}-\mathrm{BL}: \mathrm{HCMV}-\mathrm{KM})=0.00001$ ) (Figure 2B). Among the tumor suppressor genes, TP53, VHL, Rb, and TSC1 expression was mainly increased in HMECs infected with HCMV-BL compared to HCMV-TB40/E (p-value (TP53- HCMV-BL: HCMV$\mathrm{TB} 40 / \mathrm{E})=0.04 ; p$-value $(\mathrm{SMAD} 4-\mathrm{HCMV}-\mathrm{BL}: \mathrm{HCMV}-\mathrm{TB} 40 / \mathrm{E})=0.82 ; p$ value (VHL- HCMV-BL: HCMV-TB40/E) $=0.27$; p-value $(\mathrm{Rb}-\mathrm{HCMV}-\mathrm{BL}$ : HCMV-TB40/E) $=0.04 ; p$-value $($ TSC1- HCMV-BL: HCMV-TB40/E) $=0.51)$, HCMV-FS ( $p$-value (TP53- HCMV-BL: HCMV-FS) $=0.04 ; p$-value (SMAD4- HCMV-BL: HCMV-FS) $=0.12 ; p$-value (VHL- HCMV-BL: HCMV-FS $)=0.51 ; p$-value $(\mathrm{Rb}-\mathrm{HCMV}$-BL: HCMV-FS $)=0.04 ; p$-value (TSC1- HCMV-BL: HCMV-FS) 0.51 ), and HCMV-KM ( $p$-value ${ }_{\text {(TP53- }}$ HCMV-BL: HCMV-KM) $=0.04$; $p$-value $(\mathrm{SMAD} 4-\mathrm{HCMV}-\mathrm{BL}: \mathrm{HCMV}-\mathrm{KM})=$ $0.04 ; p$-value (VHL- HCMV-BL: HCMV-KM) $=0.12$; p-value (Rb- HCMVBL: HCMV-KM) $=0.04 ; p$-value $($ TSC1- HCMV-BL: HCMV-KM) $=0.12$ ) (Figure 3B). In HMECs infected with HCMV-SC, the tumor suppressor genes (TP53, VHL, TSC1) were slightly expressed, similarly in HMECs infected with HCMV-FS (Supplementary Figure 2C). The expression of TP73, a tumor suppressor gene, was markedly increased in HMECs infected with low-risk HCMV strains (KM and SC) and to a lower extent with FS and TB40/E strains compared to HMECs infected with high-risk BL strain (Figure 3B and Supplementary Figure 2C).

We then analyzed the individual expression of genes involved in cell proliferation namely STAT3, cyclin D1 (CCND1), and Ki67 antigen (MKI67). Interestingly, the STAT3/Cyclin D1 axis is activated in several cancer types including breast cancer (34). Values for individual genes were pooled to constitute the overall expression of the genes involved in cell proliferation group (Figure 2C). The expression of "proliferation" genes all together was 2.81 times more upregulated in the HMECs infected with the BL strain compared to HMECs infected with the TB40/E strain ( $p$-value (HCMV-BL: HCMV-TB40/E) $=0.05$ ) (Figure 2C). While the expression of "proliferation" genes was almost unchanged in HMECs infected with HCMV-FS compared to uninfected HMECs ( $p$-value (HCMV-BL: HCMV-FS) $=$ 0.005), it was downregulated in HMECs infected with HCMV- 

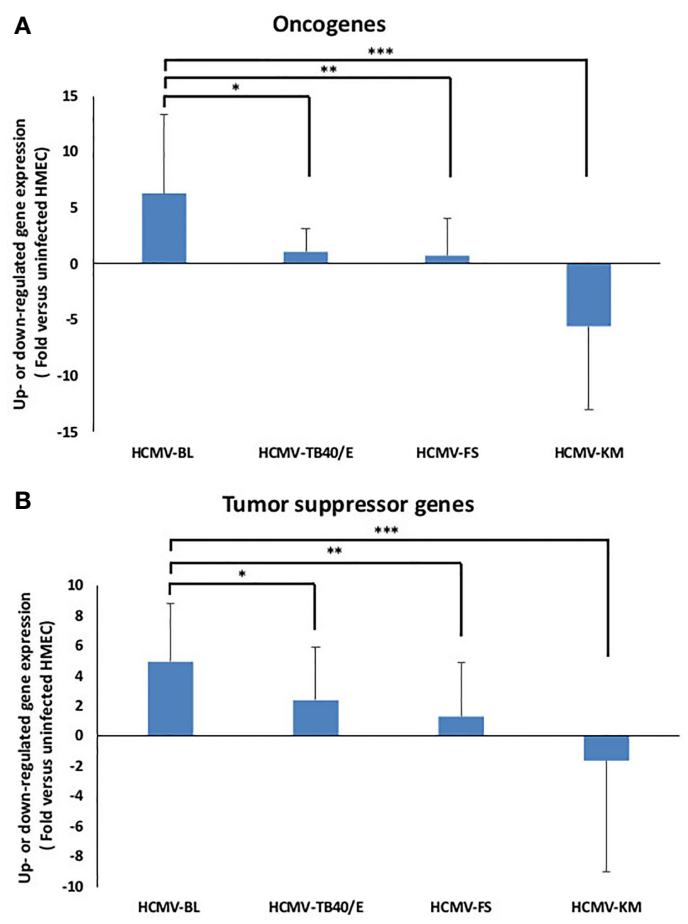

C

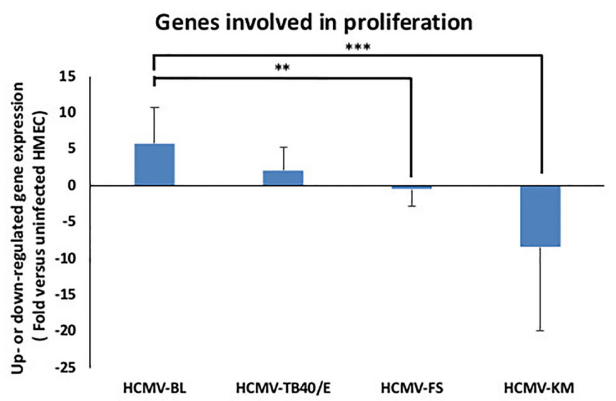

D

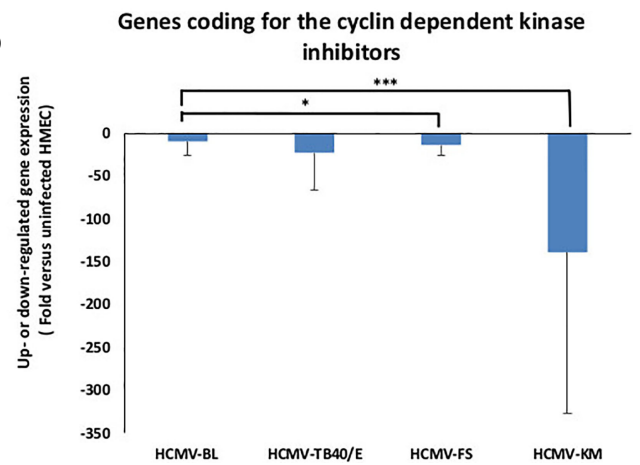

FIGURE 2 | Bulk analysis of the transcriptome differences in oncogenes, tumor suppressor genes, genes involved in cell proliferation and genes coding for cyclin dependent kinase inhibitors in HMECs infected with low-risk and high-risk HCMV strains. (A) Assessment of the bulk oncogene expression (based on MYC, FOS, KRAS, JUN, and E2F1 analysis). (B) Assessment of the bulk expression of tumor suppressor genes (based on TP53, TP73, SMAD4, VHL, RB, TSC1, and MDM2 analysis). (C) Assessment of the bulk expression of genes involved in cell proliferation (based on STAT3, CCND1, and MKI67 analysis). (D) Assessment of the bulk expression of genes coding for the cyclin-dependent kinase inhibitors (based on CDKN1A, CDKN2A, CDKN2B, and CDKN3 analysis). The gene expression was measured in HMECs infected with HCMV-BL, HCMV-TB40/E, HCMV-FS and HCMV-KM (MOI = 1) and compared to uninfected HMECs. Histograms represent mean \pm SD of three independent experiments. $p$-values were determined by Wilcoxon test. ${ }^{*} p$-value $<0.05 ;{ }^{* *} p$-value $<0.01$; ${ }^{* \star *} p$-value $<0.001$.

KM (p-value (HCMV-BL: HCMV-KM) $=0.0007)$ (Figure 2C). The STAT3 gene expression was up-regulated in HMECs infected with HCMV-BL in contrast to HMECs infected with HCMVTB40/E ( $p$-value (HCMV-BL: HCMV-TB40/E) $=0.04)$ and HCMV-FS ( $p$-value (HCMV-BL: HCMV-FS) $=0.04)$ noting that it was substantially down-regulated in HMECs infected with HCMVKM (p-value (HCMV-BL: HCMV-KM) $=0.04)$ (Figure 3C). The gene expression of cyclin D1 was up-regulated in HMECs infected with HCMV-BL compared to uninfected controls. In HMECs infected with HCMV-BL, cyclin D1 gene expression was 2 times more increased when compared to HMECs infected with HCMV-TB40/E (p-value (HCMV-BL: HCMV-TB40/E) $=0.12)$. In contrast, the expression of cyclin D1 was almost unchanged in HMECs infected with HCMV- FS compared to uninfected HMECs (p-value (HCMV-BL: HCMV-FS) $=0.12$ ), and downregulated with in HMECs infected with HCMV-KM ( $p$-value $($ HCMV-BL: HCMV-KM) $=0.04)($ Figure $3 C)$. In agreement with the highest expression of cyclin D1 in HMECs infected with HCMV$\mathrm{BL}$ and the lowest expression in HMECs infected with HCMVKM strain, we observed the highest expression of the proliferation marker Ki67 gene (MKI67) in the former and very low expression in the latter (3-fold versus 0.6 -fold increase compared to uninfected cells) (Figure 3C). HMECs infected with HCMV-SC showed a low expression of the genes involved in proliferation (CCDN1, MKI67) likewise in HMECs infected with the low-risk strains (Supplementary Figure 2C). We also assessed the expression of CDK inhibitors CDKN1A, CDKN2A, CDKN2B and CDKN3. Values for individual genes were pooled to constitute the overall expression of CDK inhibitors group (Figure 2D). The expression of CDK inhibitors genes was slightly decreased in HMECs infected with HCMV-BL compared to the other three strains TB40/E ( $p$-value (HCMV-BL: HCMV-TB40/E) $=0.81), \mathrm{FS}$ ( $p$-value (HCMV-BL: $^{-}$ HCMV-FS $)=0.02)$ and KM $(p$-value $($ HCMV-BL: HCMV-KM) $=0.001)$ (Figures 2D, 3D). HMECs infected with HCMV-SC showed a minimal expression of the genes coding for the cyclin dependent kinase inhibitors (Supplementary Figure 2C).

\section{HMECs Infected With Low-Risk and High- Risk HCMV Clinical Strains Display Distinct Expression of Cell Survival, Cell Adhesion, Angiogenesis, and EMT Genes}

The expression of genes involved in cell survival, such as $A K T$, was upregulated in HMECs infected with BL, and TB40/E strains unlike in HMECs infected with FS and KM strains ( $p$-value (HCMV-BL: HCMV-FS) $=0.12$ and p-value (HCMV-BL: HCMV-KM) $=0.04$ ) (Figure 4A). In addition, REL was upregulated more in 


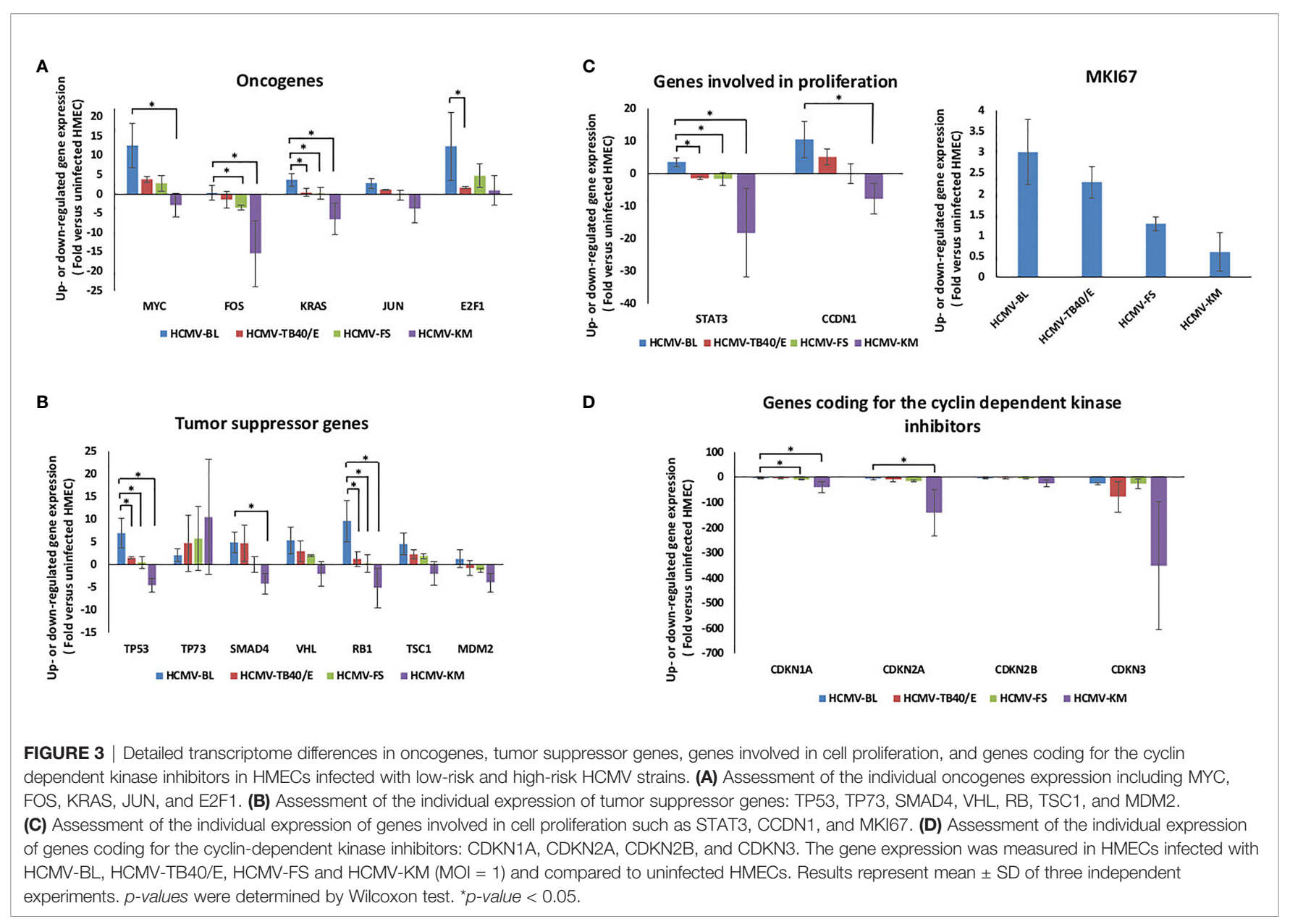

TABLE 1 | The fold change of several oncogenes' expression in HMECs infected with HCMV-BL and HCMV-FS versus uninfected HMECs.

Gene expression in HMECs infected with(Fold versus uninfected HMECs)

\begin{tabular}{lcc}
\hline Gene & HCMV-BL & HCMV-FS \\
\hline KITLG & $5.04^{*}$ & 1.93 \\
MYB & 11.10 & 6.15 \\
RARA & $74.67^{*}$ & 36.28 \\
ROS1 & $29.50^{*}$ & 14.04 \\
RUNX1 & 3.49 & 2.45
\end{tabular}

*Upregulated gene expression at least two times more in HMEC infected with HCMV-BL compared to HCMV-FS.

HCMV-BL than in HCMV-TB40/E ( $p$-value (HCMV-BL: HCMV-TB40/ E) $=0.04)$ and HCMV-FS ( $p$-value $(\mathrm{HCMV}-\mathrm{BL}: \mathrm{HCMV}-\mathrm{FS})=0.04)$ and was downregulated in HCMV-KM ( $p$-value (HCMV-BL: HCMV-KM) $=0.04)$ (Figure 4A). The expression of genes involved in DNA reparation (ATM and MLH) was downregulated in HMECs infected with HCMV-TB40/E, FS and KM compared to HMEC infected with HCMV-BL ( $p$-value (ATM-HCMV-BL: HCMV-KM) $=0.04$ and $p$-value (ATM-HCMV-BL: HCMV-FS) $=0.04$ ) (Figure 4B). The gene expression of the molecules involved in cell adhesion such as Ecadherin (CDH1) and beta-catenin (CTNNB1) was minimal in HMECs infected with the four strains (BL, TB40/E, FS and KM) noting that it was mostly downregulated in HCMV-KM ( $p$-value (CDH1-HCMV-BL: HCMV-KM) $=0.04$ and (p-value (CTNNB1-HCMV-BL:
HCMV-KM) $=0.04)$ (Figure 4C). The genes involved in angiogenesis regulation S100A4 and EGF were highly expressed in HMECs infected with HCMV-BL compared to HMECs infected with HCMV-FS and HCMV-KM ( $p$-value (S100A4-HCMV-BL: HCMVкм) $=0.1 ; p$-value $($ S100A4-HCMV-BL: HCMV-FS $)=0.5 ; p$-value ${ }_{(\mathrm{S} 100 \mathrm{~A} 4}-$ HCMV-BL: HCMV-TB40/E) $=0.1 ; p$-value (EGF-HCMV-BL: HCMV-KM) $=0.2$; $p$-value (EGF-HCMV-BL: HCMV-FS) $=0.05 p$-value (EGF-HCMV-BL: HCMVTв40/E) $=0.5$ ) (Figure 4D). Furthermore, the expression of genes involved in proteolysis including matrix metallopeptidase 9 (MMP9) and cathepsin D (CTSD) was 7-fold and 2.4-fold higher respectively, in HMECs infected with HCMV-BL compared to HMECs infected with HCMV-FS (Supplementary Table 4). The expression of the EMT markers for instance the 


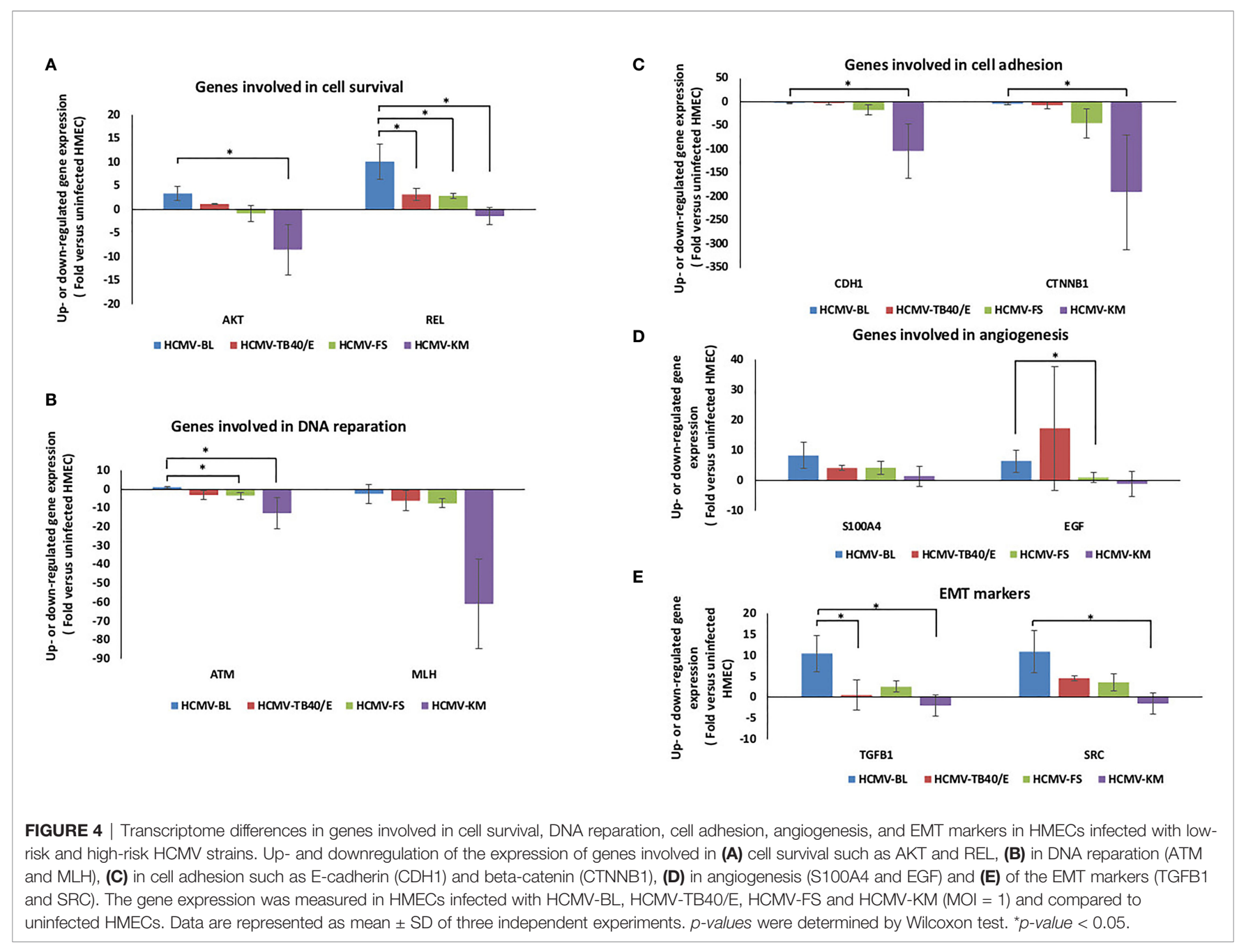

transforming growth factor-beta 1 (TGFB1) and the protooncogene tyrosine-protein kinase (SRC) was enhanced in HMECs infected with HCMV-BL compared to cells infected with HCMV-TB40/E ( $p$-value (TGFB1- HCMV-BL: HCMV-TB40/E) = 0.04; $p$-value (SRC- HCMV-BL: HCMV-TB40/E) $=0.27)$, FS $(p$-value (TGFB1- HCMV-BL: HCMV-FS) $=0.12$; $p$-value (SRC- HCMV-BL: HCMV-FS) $=0.12)$ and $\mathrm{KM}$ (p-value (TGFB1- HCMV-BL: HCMV-KM) $=0.04 ; p$ value (SRC- HCMV-BL: HCMV-KM) $=0.04$ ) (Figure 4E). In HMECs infected with HCMV-SC, the genes involved in cell survival (AKT, REL), DNA reparation (MLH), and angiogenesis (S100A4) as well as the EMT marker (SRC) were regulated in a similar manner compared to HMECs infected with the FS strain (Supplementary Figure 2C).

\section{Mammosphere Formation in HMECs Infected With Low-risk and High-Risk HCMV Clinical Strains}

We previously observed that the high-risk strains (HCMV-BL and DB) favor the appearance of stemness in chronically infected HMECs and CMV-transformed HMECs (CTH cells) (21), further suggesting that HCMV strains that trigger cellular transformation could also promote stemness. Therefore, HMECs were infected with the high-risk strain HCMV-BL, the low-risk strains HCMV-FS and HCMV-KM, and the prototypic HCMV-TB40/E. At day 1 post-infection, mammosphere formation assay was performed as previously described (35, 36). When we challenged these HCMV-infected cultures to form mammospheres, we detected numerous mammospheres in HCMV-BL infected HMECs as previously reported for HCMV-DB infected cells ( $p$-value (HCMV-BL: HMEC UI) $=0.03$ ) (37), whereas HCMV-TB40/E displayed a limited number of mammospheres ( $p$-value (HCMV-TB40/E: HMEC UI) $=0.07$ ); only few mammospheres were observed in HMECs infected with HCMV-FS and HCMV-KM (Figure 5). The breast cancer cell lines MCF7 and MDA-MB231 generated mammospheres as previously reported (37) (Figure 5). Moreover, few mammospheres were observed in uninfected HMECs as previously described (38) (Figure 5). Altogether, we noticed a preferential formation of mammospheres in HMECs infected with the high-risk HCMV-BL strain in opposition to the low-risk HCMV-TB40E (p-value (HCMV-BL: HCMV-TB40/E) $=0.03$ ), HCMV- 
A
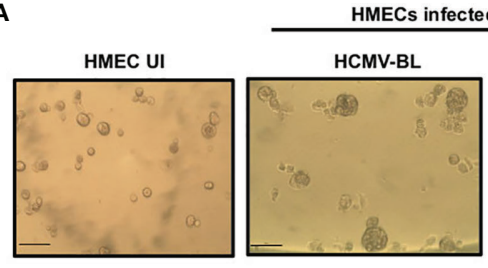

HCMV-FS
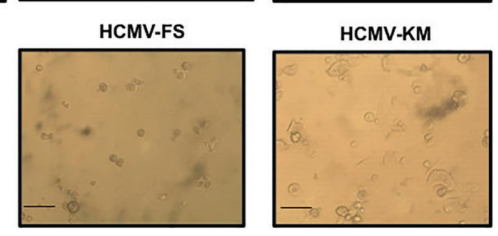

MCF7

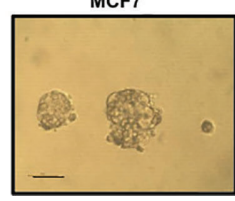

MDA-MB231

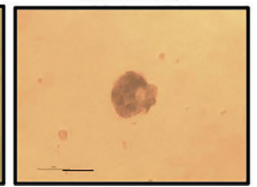

B

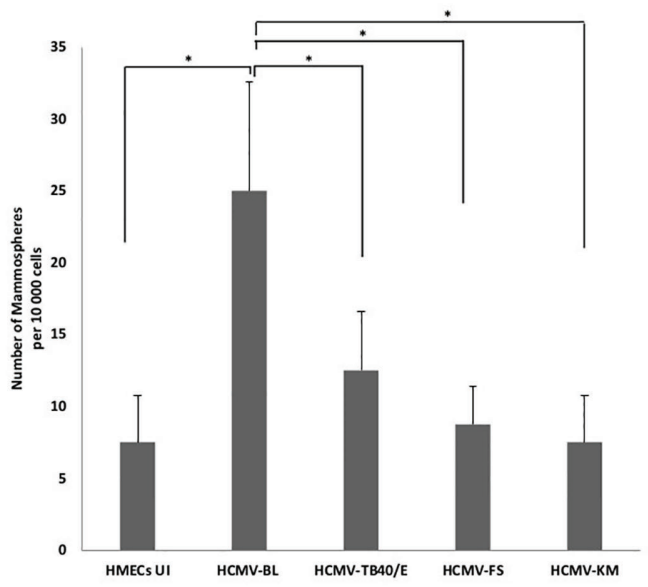

FIGURE 5 | Mammosphere formation in HMEC cultures infected with low-risk (HCMV-TB40/E, FS, and KM) and high-risk (HCMV-BL) strains. (A) Mammospheres

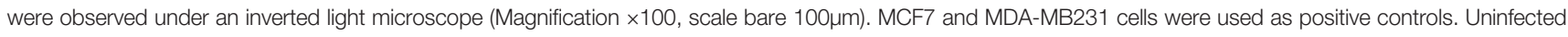
HMECs were used as a negative control. (B) The histogram represents the number of mammospheres per 10000 cells in uninfected and infected HMECs. Data are represented as mean $\pm \mathrm{SD}$ of three independent experiments. $p$-values were determined by Wilcoxon test. ${ }^{*} p$-value $<0.05$.

FS ( $p$-value (HCMV-BL: HCMV-FS) $=0.03)$ and HCMV-KM ( $p$-value $($ HCMV-BL: HCMV-KM) $=0.03)$ strains.

\section{Side-By-Side Comparison of the Prototypic Strain TB40/E and the High- Risk DB Strain for Some Specific Phenotypic and Functional Traits}

Since we observed limited mammospheres in HMECs infected with HCMV-TB40/E (Figure 5) and a slight increase in telomerase activity which has been previously reported (20), we decided to compare side by side the activation of oncogenic molecular pathways in HMECs infected with HCMV-TB40/E and the high-risk HCMV-DB strain. The productive HCMV-DB and TB40/E infection was confirmed in HMECs where both confocal microscopy imaging and flow cytometry data showed the IE1 staining (Figures 1A, B). At the protein expression level (day 3 post-infection), $\mathrm{Rb}$ was diminished whereas $\mathrm{pRb}$ was highly expressed in HMEC-HCMV-DB ( $p$-value (HCMV-DB: HMEC-UI) $=0.04$ ) and to a lesser extent in HMEC-HCMVTB40/E (p-value (HCMV-TB40/E: HMEC-UI) $=0.04$, p-value ${ }_{(\mathrm{HCMV}-}$ $\mathrm{TB} 40 / \mathrm{E}: \mathrm{HCMV}-\mathrm{DB})=0.8)($ Figures 6A, B). At the same time point, pSTAT3 expression was higher in HMEC-HCMV-DB ( $p$-value $($ HCMV-DB: HMEC-UI) $=0.03)$ compared to HMEC-HCMV-TB40/E $(p$-value $(\mathrm{HCMV}-\mathrm{TB} 40 / \mathrm{E}:$ HMEC-UI) $=0.06)$ and STAT3 expression was further increased in HMEC-HCMV-DB compared to HMEC-HCMV-TB40/E (Figures 6A, C). Further, Akt expression was highly increased in HMEC-HCMV-DB ( $p$ value (HCMV-DB: HMEC-UI) $=0.03$ ), compared to HMECHCMV-TB40/E at day 3 post-infection ( $p$-value (HCMV-TB40/E: HMEC-UI) $=0.06$ ), and an increase in its phosphorylation on both serine 473 and threonine 308 in HMEC-HCMV-DB was observed (Figures 6A, D). At day 1 post-infection, cyclin D1 expression was increased in HEMC-HCMV-DB ( $p$-value ${ }_{\text {HCMV- }}$ $\mathrm{DB}:$ HMEC-UI $)=0.03)$ compared to HMEC-HCMV-TB40/E $(p-$ value $\left._{(\mathrm{HCMV}-\mathrm{TB} 40 / \mathrm{E} \text { : HMEC-UI) }}=0.06\right)($ Figures 6A, E). The protein expression of ATM was elevated in HMECs infected with HCMV-DB in comparison to HCMV-TB40/E (Figures 6A, F). Our results indicate that the HCMV-TB40/E strain does not induce significant activation of the molecular oncogenic pathways, especially when compared to the high-risk strain HCMV-DB, and therefore should be classified as a low-risk HCMV strain consistent with the absence of colony formation in soft agar in HMEC cultures seeded with TB40/E.

\section{Detection of HCMV in Breast Cancer Biopsies}

To assess the impact of HCMV strains on cellular gene expression in vivo, Ki67 expression was quantified by RTqPCR in breast tumor biopsies, including luminal and basallike tumors compared to healthy breast tissues. First, we measured the percentage of healthy and tumor biopsies with low $(<30)$, intermediate $(30-100)$ or high $(>100)$ Ki67 mRNA expression (Figures 7A, B). Basal-like biopsies accounted for the highest percentage of biopsies with high Ki67 mRNA expression level (\% of basal-like: $44 \%$ ) compared to healthy $(2 \%)$ and luminal biopsies (20\%) ( $p$-value ${ }_{\text {(High Ki67 - Basal : Healthy) }}=0.02$ ) (Figure 7A). To further study the role of HCMV associated with Ki67 transcript expression, we assessed the presence of HCMV in the biopsies by quantifying IE1 and UL69 genes using qPCR (21, 30). In HCMV-positive biopsies, basal-like biopsies showed the 
A

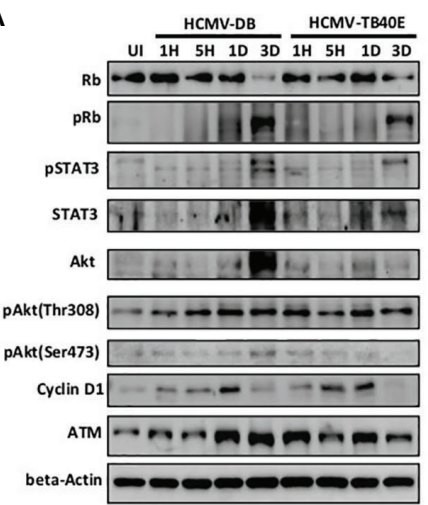

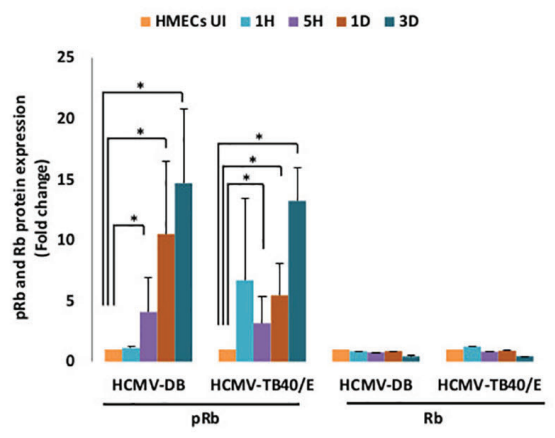

C

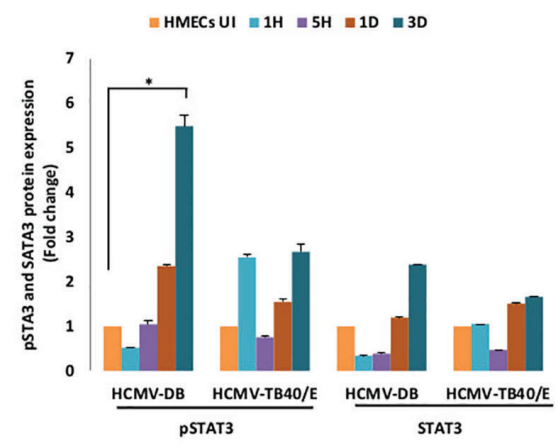

D

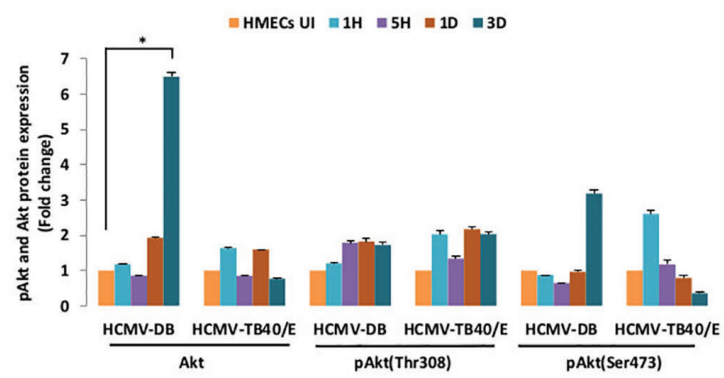

E

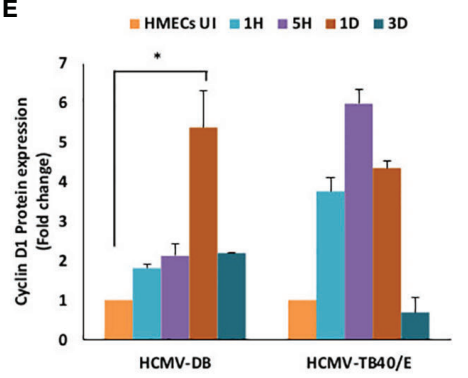

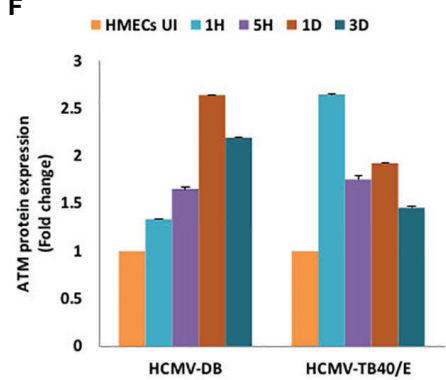

FIGURE 6 | Distinct activation of oncogenic pathways in HMECs infected with the high-risk DB strain and the prototypic TB40/E strain. (A) Immunoblotting data of Rb, pRb, STAT3, pSTAT3, Akt, pAkt-Thr308, pAkt-Ser473, cyclin D1 and ATM in uninfected HMECs and HMECs infected with HCMV-DB and HCMV-TB40/E $(\mathrm{MOI}=1)$ at $1 \mathrm{H}, 5 \mathrm{H}, \mathrm{D} 1$ and $\mathrm{D} 3$ post-infection. $\beta$-actin was used as loading control. Results are representative of three independent experiments. Histograms representing (B) Rb, pRb, (C) STAT3, pSTAT3 (D) Akt, pAkt-Thr308, pAkt-Ser473, (E) cyclin D1, and (F) ATM expression in HMECs infected with HCMV-DB and HCMV-TB4O/E $(\mathrm{MOI}=1)$ at $1 \mathrm{H}, 5 \mathrm{H}, \mathrm{D} 1$ and D3 post-infection; protein expression is shown based on quantification by ImageJ 1.40 software. Data are represented as mean \pm SD of three independent experiments. $p$-values were determined by Wilcoxon test. ${ }^{*} p$-value $<0.05$.

highest percentage of biopsies with high levels of Ki67 mRNA (40\%) in contrast to the healthy $(0 \%)$ and luminal (0\%) biopsies (Figure 7B). Second, we assessed the absolute level of Ki67 mRNA per biopsy of healthy tissues, luminal and basal-like tumors taking into account the presence or absence of HCMV (Figure 7C). Healthy biopsies showed low absolute levels of Ki67 mRNA compared to tumor biopsies independent of HCMV status (presence or absence) $(p$-value $=0.02)$. In tumor biopsies, the absolute level of Ki67 mRNA was enhanced by 2.5-fold in HCMV-positive biopsies compared to HCMVnegative biopsies ( $p$-value $=0.2$ ). In HCMV-positive biopsies, the absolute level of Ki67 mRNA in basal tumors was enhanced by 31.45 -fold compared to luminal tumors $(p$-value $=0.17$ ) (Figure 7C). The absolute level of Ki67 mRNA was shown separately for each biopsy where cutoff lines were set to differentiate between low and high Ki67 expression and allow a closer look on the variability of Ki67 mRNA expression per sample (Supplementary Figure 3). Thus, when we independently measured the absolute level of Ki67 mRNA in each HCMV-positive biopsy, we detected two basal biopsies (biopsies \#20 and \#21) showing the highest absolute levels of Ki67 mRNA with 315 and 4015-fold increase versus the mean value of healthy tissue, respectively (Figure 7D). Meanwhile, much lower absolute levels of Ki67 mRNA were detected in the other HCMV-positive tumor and healthy biopsies. Low absolute levels of Ki67 mRNA were detected in HCMV-positive luminal biopsies (less than 30-fold increase versus mean value of healthy tissue) except for one biopsy (biopsy \#15 with 108-fold increase) (Figure 7D).

Within our data, we were able to identify two biopsies, namely biopsies \#20 and \#21 (Figure 7D, arrows), that could harbor highrisk HCMV strains. Given the total number of biopsies, we might not be able to properly identify all the high-risk strains, which effect might be partially masked in the overall HCMV-positive biopsies. To confirm the high-risk status of biopsies \#20 and \#21, we isolated the strains for each biopsy and used it to infect HMECs (Supplementary Figure 4A). After few weeks in culture, we observed dense structures (Supplementary Figure 4B), similar to the morphology of the previously reported CTH cells $(20,21)$. These emerging structures were not present in HMECs infected with lowrisk strains that were isolated from other biopsies in which we observed cell lysis. Overall, we identified two high-risk strains $(n=2$; $15 \%$ ) out of the HCMV-positive biopsies ( $n=13)$; upon assessing the proportion of high-risk strain within tumor biopsies only, we identified two high-risk strains $(n=2 ; 20 \%)$ out of the total HCMV-positive tumors $(n=10)$. 


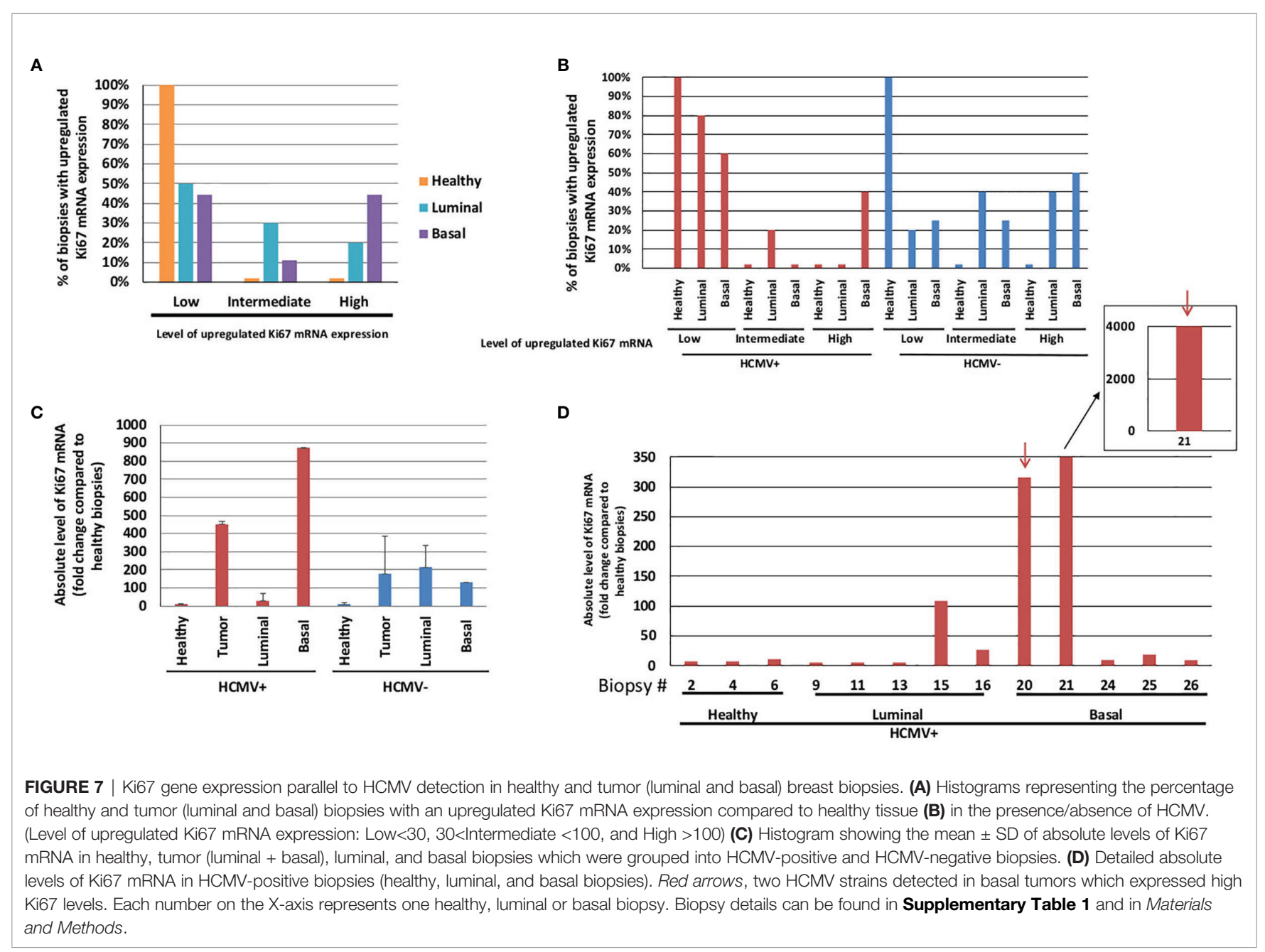

Finally, using twenty different breast tumor biopsies, we aimed at evaluating the value of Ki67 expression as an index for increased proliferation in breast cancer. Ki67 mRNA and protein levels were measured by RT-qPCR and IHC, respectively, on the same biopsy sample. A correlation was observed between Ki67 protein and mRNA levels (Supplementary Figure 5). This data indicates that Ki67 mRNA expression could be considered as a reliable marker for the measurement of increased proliferation in breast cancer.

\section{DISCUSSION}

We previously isolated the two clinical isolates, HCMV-DB and HCMV-BL, which in primary human mammary epithelial cells (HMECs) promoted oncogenic molecular pathways, established anchorage-independent growth in vitro, and produced tumorigenicity in mice models $(20,21)$, therefore named highrisk oncogenic strains. In contrast, other clinical HCMV strains such as HCMV-FS, KM and SC did not trigger such traits (21), therefore named low-risk oncogenic strains. TB40/E strain did not induce significant activation of the molecular oncogenic pathways in HMECs, especially when compared to the highrisk strain HCMV-DB, and therefore should be classified as a low-risk HCMV strain consistent with the absence of colony formation in soft agar. In contrast to the other HCMV low-risk strains, HCMV-KM was isolated from a two-year old infant with HCMV congenital infection which might explain the different observed transcriptome profile compared to other low-risk HCMV strains (39). Our results indicate the capacity of the six tested HCMV clinical strains to replicate efficiently in HMEC cells allowing the comparison of their transcriptome profile. The transcriptome of HMECs infected with HCMV-BL strain exhibits a pro-oncogenic cellular environment with enhanced expression of several oncogenes, proliferation markers, cell survival genes, and EMT traits (Figure 8). Moreover, these pro-oncogenic characteristics were accompanied with stemness traits as assessed in vitro by mammosphere formation. In contrast, based on the transcriptome analysis, the HCMV-FS, $\mathrm{KM}$ and SC strains were less prone to favor an oncogenic environment (Figure 8). The prototypic TB40/E strain displays mostly low-oncogenic traits as assessed by its proteomic profile in infected HMECs (Figure 8). Finally, we observed a potential link between the presence of HCMV in the breast tumor, 


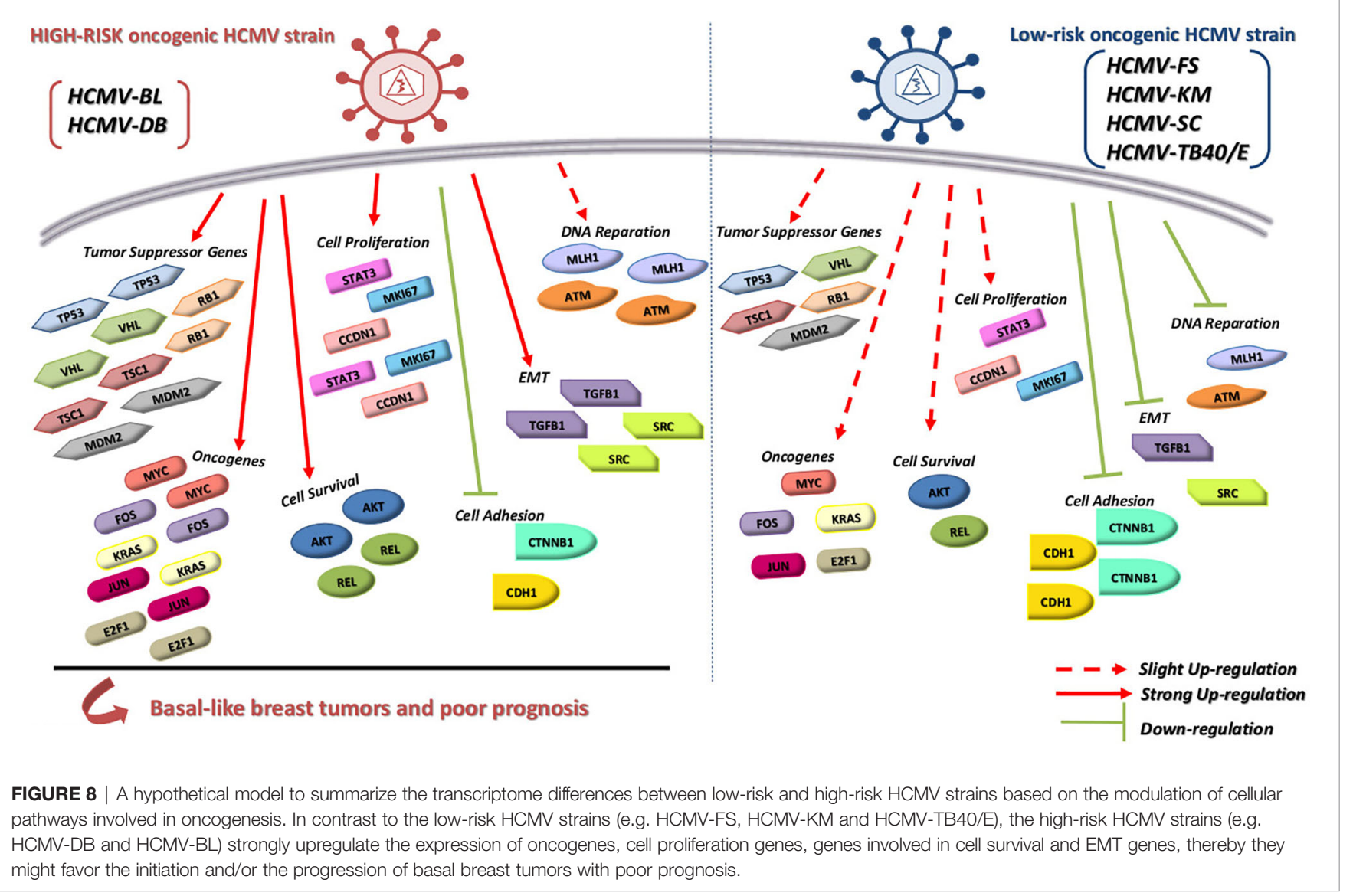

especially the basal tumors, and high levels of Ki67 mRNA further suggesting the presence of high-risk HCMV strains in some of the basal tumors. Altogether, the transcriptome profiles of HCMV-infected HMECs and the analysis of Ki67 mRNA levels in breast tumors might allow for the classification of high and low-risk HCMV strains based on their oncogenic potential.

Although HCMV clinical isolates display a broad cellular tropism, the growth of laboratory HCMV strains is restricted to fibroblasts (16). Furthermore, several clinical HCMV strains can generate distinct inflammatory (M1) and immunosuppressive (M2) macrophage polarization after infection of blood monocytes and tissue macrophage and can establish latency in these latter $(17,23,25,40)$. The preferential tropism of clinical HCMV strains might favor their long-lasting low-level viral replication in HMECs and ultimately promote breast cancer (41-43). Taking into account its tropism for mammary epithelial cells and its transcriptome oncogenic environment, the HCMV$\mathrm{BL}$ strain is close to the previously described HCMV-DB strain (37). Based on their transforming capacity in vitro (soft agar), the activation of oncogenic and proliferation pathways including Myc and Ki67 overexpression, and the development of tumors when injected in NSG mice $(20,21)$ the HCMV-DB and HCMVBL strains are classified as high-risk strains.

Inactivation of tumor suppressor proteins such as retinoblastoma protein $(\mathrm{Rb})$ and $\mathrm{p} 53$ and activation of pro- oncogenes such as Myc and Ras are critical events involved in HMEC cells transformation and immortalization $(44,45)$ and are known to favor breast cancer progression (46). Intriguingly, the expression of p53 was 7-fold higher in HMECs infected with high-risk strain HCMV-BL compared to uninfected HMECs. Enhanced expression of p53 has been described in human mammary epithelial cells infected with HCMV-DB $(20,37)$ and in fibroblasts infected with numerous HCMV strains $(47,48)$. To explain the functional inactivation of p53 needed for cell transformation, a potential inhibitory effect of IE2 on p53 transcriptional activity has been previously reported (49). In addition, we previously described p53 protein binding to IE2 in HCMV-DB infected HMECs, leading to its functional inactivation parallel to p53 transcript upregulation $(20,37)$. A similar mechanism might be in action in HMECs infected with HCMV-BL.

Likewise, the expression of $\mathrm{Rb}$ gene is increased in HMECs infected with HCMV-BL by 10-fold compared to the uninfected HMECs. Several small DNA tumor viruses such as adenovirus, human papillomavirus, and polyomavirus were described to abolish the function of $\mathrm{Rb}$ protein (50). In addition, it was reported that $\mathrm{Rb}$ inactivation mediated by viral proteins through the E2F transcriptional factor is necessary for viral replication and mammalian cells transformation (50,51). Also, the inactivation of $\mathrm{Rb}$ protein could be caused by posttranslation phosphorylation (52). For HCMV, manipulation of 
the cell cycle through $\mathrm{Rb}$ inactivation could favor cell transformation (53). First, the viral protein pp71 degrades the hypophosphorylated $\mathrm{Rb}$ protein earlier during infection, then $\mathrm{Rb}$ protein reaccumulates later during infection (54-56). Second, the viral protein UL97 phosphorylates Rb protein on several CDK target sites and deactivates it. Third, the viral proteins IE1 and IE2 were described to activate E2F-responsive promoters and most probably induce $\mathrm{Rb}$ inactivation (57-59). Interestingly, parallel to enhanced $\mathrm{Rb}$ gene expression we observed high levels of E2F1 transcript in HMECs infected with the high-risk $\mathrm{BL}$ strain. Thus, the inactivation of both $\mathrm{p} 53$ and $\mathrm{Rb}$ proteins is mandatory for cell transformation in HCMV-BL and HCMVDB infected HMECs which might explain that even under enhanced p53 and $\mathrm{Rb}$ gene expression, their protein activity will be abolished.

In agreement with our previous results (21), the overexpression of Myc and KRas could participate in the transformation process of HMEC cells infected with HCMV-BL. In addition, the enhanced expression of other oncogenes such as Jun and E2F1 observed in HMECs infected with HCMV-BL could stimulate transformation $(60,61)$. Interestingly, the viral proteins IE1 and IE2 induce Myc activation (62). We observed previously a similar activation of Myc and Ras protein and gene expression in HMECs infected with the HCMV-DB strain $(20,37)$.

Furthermore, one of the mechanisms described to transform HMECs is the link of Myc overexpression to PI3K/Akt pathway activation $(63,64)$. We observed mostly enhanced expression of Akt gene in HMECs infected with HCMV-BL compared to HCMV-FS, $\mathrm{KM}$ and TB40/E in agreement with our previous results (21), and an upregulation of serine-473 phosphorylation of Akt in HMECs infected with HCMV-DB compared to HCMV-TB40/E. The $\mathrm{PI} 3 \mathrm{~K} /$ Akt activation in HCMV infected cells is in line with previous findings (65). In addition, several studies have pointed the role of REL in cell growth and oncogenesis (66). The overexpression of REL was associated with cell transformation in vitro and/or in vivo. Epstein-Barr virus (EBV) activates REL/NF-kB during malignant transformation (67). Thus, the overexpression of REL in HMECs infected with HCMV-BL is consistent with its high oncogenic potential.

In agreement with the HCMV-DB transcriptome study (37), an enhanced expression of proliferation markers such as STAT3, cyclin D1, and especially Ki67 was observed in HMEC cells infected with HCMV-BL but not with HCMV- FS, KM, SC and TB40/E. The proliferation and invasion of several cancers could be explained by STAT3 activation and cyclin D1 overexpression (68). Moreover, the overexpression of pSTAT3 and STAT3 at day 3 post-infection was observed mainly in HMECs infected with HCMV-DB, but to a lesser extent with HCMV-TB40/E. These findings confirm that the high-risk HCMV strains such as $\mathrm{BL}$ and $\mathrm{DB}$ have high proliferation capacities compared to the low-risk HCMV strains, such as TB40/E, FS, KM and SC.

Studies have reported that ataxia telangiectasia mutated (ATM) is required for efficient viral replication of numerous viruses including HCMV, HSV-1, HPV, and HIV (69-73). Indeed, HCMV infection leads to host DNA damage responses (DDRs) with activation of the DNA reparation pathway (74-76).
Enhanced expression of ATM gene was observed in infected HMECs with HCMV-BL and with HCMV-DB as reported previously (37). Lower levels of ATM expression were detected in HMECs infected with HCMV-TB40/E, FS, and KM. The enhanced expression of ATM can promote genome instability resulting in cellular transformation. MLH expression is necessary for efficient HSV-1 viral replication (77) and its enhanced expression has been linked to high levels of genomic instability, cancer progression, and aggressiveness $(78,79)$. Likewise, the overexpression of MMP9 and CTSD observed in HMECs infected with HCMV-BL was associated with the most aggressive subtype of breast cancer (80), tumor cell invasion, and metastasis (81-84).

The expression of genes involved in EMT such as the protooncogene tyrosine-protein kinase Src (85) and the transforming growth factor-beta 1 (TGFB1) $(86,87)$ was higher in HMECs infected with HCMV-BL than with HCMV-TB40/E, FS, KM and SC. Our findings are in accordance with our previous analysis of HCMV-DB transcriptome (37).

Altogether the transcriptome analysis of HMECs infected with the high-risk strain HCMV-BL underlines the potential capacity of the emergence of cancer stem cells (CSCs) compared to the HMECs infected with low-risk strains (HCMV-FS, KM and SC). In line with our transcriptome data and with the previously reported enhanced mammosphere formation in HMECs infected with HCMV-DB (37), we observed that the number of mammospheres detected in HMECs infected with HCMV-BL was 2-fold, 2.8-fold and 3-fold higher compared to HMECs infected with HCMV-TB40/E, HCMV-FS and KM, respectively

The distinct oncogenic potential of HCMV strains observed at the transcriptome level in vitro was then assessed in vivo in breast tumors. The nuclear protein Ki67 is not required only for cell proliferation in tumor but also for tumor initiation, growth, and metastasis. Moreover, Ki67 inactivation leads to transcriptome remodeling that alters mesenchymal-epithelial balance and suppresses stemness (88). Ki67 is highly expressed in malignant tissues with poorly differentiated tumor cells (89). High Ki67, possessing high proliferation potential, was reported to worsen the survival of triple negative breast cancer patients and to trigger quick tumor recurrence within a short period of time (90). Remarkably, enhanced expression of Ki67 gene and upregulation of Ki67 protein was observed in HMECs infected with the HCMVDB and BL strains $(20,21)$. Therefore, we assessed the link between HCMV presence and Ki67 gene overexpression in breast tumor biopsies. Basal-like biopsies revealed the highest percentage of biopsies with high levels of Ki67 mRNA compared to the healthy and luminal biopsies. Only tumor biopsies were impacted by HCMV status; the highest level of Ki67 mRNA was detected in HCMV-positive tumor biopsies compared to HCMV-negative tumor biopsies. Among the HCMV-positive biopsies, the absolute level of Ki67 was low in all healthy and most of the luminal biopsies except for one luminal biopsy (\#15 with limited increase in Ki67 absolute level). In contrast, among the tested basal-like HCMVpositive biopsies, we observed augmented absolute levels of Ki67 mRNA in two biopsies (biopsies \#20 and \#21) despite the low absolute levels of Ki67 mRNA that were detected in some biopsies. 
The HCMV strains that were isolated from biopsies \#20 and 21, transformed the freshly uninfected HMECs resulting in the appearance of CMV-transformed HMECs (CTH cells) in vitro indicating their high-risk profile (Supplementary Figure 4). Since high-risk HCMV strains strongly enhanced the expression of Ki67Ag $(20,21)$, the majority of the HCMV strains detected in the breast tumor biopsies or in the adjacent tested healthy tissues might be non-oncogenic or low-risk HCMV strains. The detection of very high expression of Ki67 mRNA in two basal tumor biopsies concomitant with the presence of HCMV suggests a potential link between high-risk HCMV strains present in the tumor tissue, highly enhanced Ki67 expression, and the initiation and/or progression of basal breast tumors. In agreement with our results, Ki67 was described as a key marker associated with poor prognosis in triple-negative breast cancer (91). We are currently studying a larger cohort of breast cancer biopsies and assessing additional "oncogenic" markers such as c-Myc to further highlight the exact role of HCMV strains in breast oncogenesis.

\section{CONCLUSION}

In conclusion, our data indicate the capacity of high-risk HCMV strains to induce a pro-oncogenic cellular environment in contrast to the low-risk strains. Similar to the HCMV-DB strain, HCMV$\mathrm{BL}$ was able to induce enhanced expression of various oncogenes, genes involved in proliferation, cell survival, DNA reparation, and EMT. Additionally, high-risk HCMV strains present stemness traits with enhanced mammosphere formation in vitro compared to the low-risk strains. In vivo, we detected the concomitant presence of a very high Ki67 gene expression in two HCMVpositive basal breast tumors, indicating a potential link between high-risk HCMV strains present in the tumoral tissue and the development of aggressive tumors of poor prognosis. The discrimination between HCMV clinical strains based on their distinct oncogenic potential will pave the way for understanding their respective role in breast cancer and open the door to new therapeutic approaches.

\section{DATA AVAILABILITY STATEMENT}

The original contributions presented in the study are included in the article/Supplementary Material. Further inquiries can be directed to the corresponding author.

\section{REFERENCES}

1. Lehmann BD, Bauer JA, Chen X, Sanders ME, Chakravarthy AB, Shyr Y, et al. Identification of Human Triple-Negative Breast Cancer Subtypes and Preclinical Models for Selection of Targeted Therapies. J Clin Invest (2011) 121:2750-67. doi: 10.1172/JCI45014

2. Hüsing A, Canzian F, Beckmann L, Garcia-Closas M, Diver WR, Thun MJ, et al. Prediction of Breast Cancer Risk by Genetic Risk Factors, Overall and by Hormone Receptor Status. J Med Genet (2012) 49:601-8. doi: 10.1136/jmedgenet-2011-100716

3. Alibek K, Kakpenova A, Mussabekova A, Sypabekova M, Karatayeva N. Role of Viruses in the Development of Breast Cancer. Infect Agent Cancer (2013) 8:32. doi: $10.1186 / 1750-9378-8-32$

\section{ETHICS STATEMENT}

The studies involving human participants were reviewed and approved by local ethics committees of Besançon University Hospital (Besançon, France) and the French Research Ministry (AC-2015-2496, CNIL n¹173545, NF-S-138 96900 n $^{\circ}$ F2015). The patients/participants provided their written informed consent to participate in this study.

\section{AUTHOR CONTRIBUTIONS}

Conceptualization, GH. Formal analysis, SH, SP, and GH. Investigation, $\mathrm{SH}, \mathrm{SP}, \mathrm{RE}, \mathrm{ZN}$, and CL. Writing-original draft preparation, $\mathrm{SH}$ and $\mathrm{GH}$. Writing-review and editing, SH, SP, RE, and GH. Visualization, SH and SP. Supervision, GH. Project administration, GH. Funding acquisition, GH. All authors contributed to the article and approved the submitted version.

\section{FUNDING}

This work was supported by grants from the University of FrancheComte (UFC) (CR3300), the Région Franche-Comte (2021-Y08292 and 2021-Y-08290) and the Ligue contre le Cancer (CR3304) to $\mathrm{GH}$. SH is recipient of a doctoral scholarship from Lebanese municipality. RE is a recipient of a doctoral scholarship from Hariri foundation for sustainable human development.

\section{ACKNOWLEDGMENTS}

We are grateful to Franck Monnien, Chloé Molimard and MariePaule Algros from the Pathology Department at the Besançon University Hospital for providing breast biopsies. We thank Dr. Davit Varron from the Mathematics Department of the University of Franche-Comte for the statistical analyses.

\section{SUPPLEMENTARY MATERIAL}

The Supplementary Material for this article can be found online at: https://www.frontiersin.org/articles/10.3389/fimmu.2021. 772160/full\#supplementary-material

4. Zur Hausen H. The Search for Infectious Causes of Human Cancers: Where and Why. Virology (2009) 392:1-10. doi: 10.1016/j.virol.2009.06.001

5. Geisler J, Touma J, Rahbar A, Söderberg-Nauclér C, Vetvik K. A Review of the Potential Role of Human Cytomegalovirus (HCMV) Infections in Breast Cancer Carcinogenesis and Abnormal Immunity. Cancers (2019) 11:1842. doi: 10.3390/cancers11121842

6. Taher C, Frisk G, Fuentes S, Religa P, Costa H, Assinger A, et al. High Prevalence of Human Cytomegalovirus in Brain Metastases of Patients With Primary Breast and Colorectal Cancers. Transl Oncol (2014) 7:732-40. doi: 10.1016/j.tranon.2014.09.008

7. Taher C, de Boniface J, Mohammad A-A, Religa P, Hartman J, Yaiw K-C, et al. High Prevalence of Human Cytomegalovirus Proteins and Nucleic Acids 
in Primary Breast Cancer and Metastatic Sentinel Lymph Nodes. PloS One (2013) 8:e56795. doi: 10.1371/journal.pone.0056795

8. El Shazly DF, Bahnassey AA, Omar OS, Elsayed ET, Al-Hindawi A, ElDesouky E, et al. Detection of Human Cytomegalovirus in Malignant and Benign Breast Tumors in Egyptian Women. Clin Breast Cancer (2018) 18: e629-42. doi: 10.1016/j.clbc.2017.10.018

9. Samanta M, Harkins L, Klemm K, Britt WJ, Cobbs CS. High Prevalence of Human Cytomegalovirus in Prostatic Intraepithelial Neoplasia and Prostatic Carcinoma. J Urol (2003) 170:998-1002. doi: 10.1097/01.ju.0000080263.46164.97

10. Harkins LE, Matlaf LA, Soroceanu L, Klemm K, Britt WJ, Wang W, et al. Detection of Human Cytomegalovirus in Normal and Neoplastic Breast Epithelium. Herpesviridae (2010) 1:8. doi: 10.1186/2042-4280-1-8

11. Baryawno N, Rahbar A, Wolmer-Solberg N, Taher C, Odeberg J, Darabi A, et al. Detection of Human Cytomegalovirus in Medulloblastomas Reveals a Potential Therapeutic Target. J Clin Invest (2011) 121:4043-55. doi: 10.1172/JCI57147

12. Bhattacharjee B, Renzette N, Kowalik TF. Genetic Analysis of Cytomegalovirus in Malignant Gliomas. J Virol (2012) 86:6815-24. doi: 10.1128/JVI.00015-12

13. Banerjee S, Wei Z, Tan F, Peck KN, Shih N, Feldman M, et al. Distinct Microbiological Signatures Associated With Triple Negative Breast Cancer. Sci Rep (2015) 5:15162. doi: 10.1038/srep15162

14. Coaquette A, Bourgeois A, Dirand C, Varin A, Chen W, Herbein G. Mixed Cytomegalovirus Glycoprotein B Genotypes in Immunocompromised Patients. Clin Infect Dis Off Publ Infect Dis Soc Am (2004) 39:155-61. doi: $10.1086 / 421496$

15. El Baba R, Herbein G. Immune Landscape of CMV Infection in Cancer Patients: From "Canonical" Diseases Toward Virus-Elicited Oncomodulation. Front Immunol (2021) 12:730765. doi: 10.3389/fimmu.2021.730765

16. Wang D, Shenk T. Human Cytomegalovirus Virion Protein Complex Required for Epithelial and Endothelial Cell Tropism. Proc Natl Acad Sci (2005) 102:18153-8. doi: 10.1073/pnas.0509201102

17. Khan KA, Coaquette A, Davrinche C, Herbein G. Bcl-3-Regulated Transcription From Major Immediate-Early Promoter of Human Cytomegalovirus in Monocyte-Derived Macrophages. I Immunol Baltim Md 1950 (2009) 182:7784-94. doi: 10.4049/jimmunol.0803800

18. Belzile J-P, Stark TJ, Yeo GW, Spector DH. Human Cytomegalovirus Infection of Human Embryonic Stem Cell-Derived Primitive Neural Stem Cells is Restricted at Several Steps But Leads to the Persistence of Viral DNA. J Virol (2014) 88:4021-39. doi: 10.1128/JVI.03492-13

19. Lepiller Q, Abbas W, Kumar A, Tripathy MK, Herbein G. HCMV Activates the IL-6-JAK-STAT3 Axis in HepG2 Cells and Primary Human Hepatocytes. PloS One (2013) 8:e59591. doi: 10.1371/journal.pone.0059591

20. Kumar A, Tripathy MK, Pasquereau S, Al Moussawi F, Abbas W, Coquard L, et al. The Human Cytomegalovirus Strain DB Activates Oncogenic Pathways in Mammary Epithelial Cells. EBioMedicine (2018) 30:167-83. doi: 10.1016/ j.ebiom.2018.03.015

21. Nehme Z, Pasquereau S, Haidar Ahmad S, Coaquette A, Molimard C, Monnien F, et al. Polyploid Giant Cancer Cells, Stemness and EpithelialMesenchymal Plasticity Elicited by Human Cytomegalovirus. Oncogene (2021) 40:3030-46. doi: 10.1038/s41388-021-01715-7

22. Geder KM, Lausch R, O’Neill F, Rapp F. Oncogenic Transformation of Human Embryo Lung Cells by Human Cytomegalovirus. Science (1976) 192:1134-7. doi: 10.1126/science.179143

23. Hargett D, Shenk TE. Experimental Human Cytomegalovirus Latency in CD14+ Monocytes. Proc Natl Acad Sci USA (2010) 107:20039-44. doi: 10.1073/pnas.1014509107

24. Noriega VM, Haye KK, Kraus TA, Kowalsky SR, Ge Y, Moran TM, et al. Human Cytomegalovirus Modulates Monocyte-Mediated Innate Immune Responses During Short-Term Experimental Latency In Vitro. J Virol (2014) 88:9391-405. doi: 10.1128/JVI.00934-14

25. Smith MS, Bentz GL, Alexander JS, Yurochko AD. Human Cytomegalovirus Induces Monocyte Differentiation and Migration as a Strategy for Dissemination and Persistence. J Virol (2004) 78:4444-53. doi: 10.1128/ jvi.78.9.4444-4453.2004

26. Zhuravskaya T, Maciejewski JP, Netski DM, Bruening E, Mackintosh FR, St Jeor S. Spread of Human Cytomegalovirus (HCMV) After Infection of Human Hematopoietic Progenitor Cells: Model of HCMV Latency. Blood (1997) 90:2482-91. doi: 10.1182/blood.V90.6.2482
27. Taylor-Wiedeman J, Sissons JG, Borysiewicz LK, Sinclair JH. Monocytes are a Major Site of Persistence of Human Cytomegalovirus in Peripheral Blood Mononuclear Cells. J Gen Virol (1991) 72(Pt 9):2059-64. doi: 10.1099/00221317-72-9-2059

28. Movassagh M, Gozlan J, Senechal B, Baillou C, Petit JC, Lemoine FM. Direct Infection of CD34+ Progenitor Cells by Human Cytomegalovirus: Evidence for Inhibition of Hematopoiesis and Viral Replication. Blood (1996) 88:127783. doi: 10.1182/blood.V88.4.1277.bloodjournal8841277

29. Sinzger C, Schmidt K, Knapp J, Kahl M, Beck R, Waldman J, et al. Modification of Human Cytomegalovirus Tropism Through Propagation In Vitro is Associated With Changes in the Viral Genome. J Gen Virol (1999) 80 (Pt 11):2867-77. doi: 10.1099/0022-1317-80-11-2867

30. Haidar Ahmad S, Al Moussawi F, El Baba R, Nehme Z, Pasquereau S, Kumar A, et al. Identification of UL69 Gene and Protein in CytomegalovirusTransformed Human Mammary Epithelial Cells. Front Oncol (2021) 11:627866. doi: 10.3389/fonc.2021.627866

31. Schmittgen TD, Livak KJ. Analyzing Real-Time PCR Data by the Comparative C(T) Method. Nat Protoc (2008) 3:1101-8. doi: 10.1038/ nprot.2008.73

32. Bustreo S, Osella-Abate S, Cassoni P, Donadio M, Airoldi M, Pedani F, et al. Optimal Ki67 Cut-Off for Luminal Breast Cancer Prognostic Evaluation: A Large Case Series Study With a Long-Term Follow-Up. Breast Cancer Res Treat (2016) 157:363-71. doi: 10.1007/s10549-016-3817-9

33. Wilkinson GWG, Davison AJ, Tomasec P, Fielding CA, Aicheler R, Murrell I, et al. Human Cytomegalovirus: Taking the Strain. Med Microbiol Immunol (Berl) (2015) 204:273-84. doi: 10.1007/s00430-015-0411-4

34. Banerjee K, Resat H. Constitutive Activation of STAT3 in Breast Cancer Cells: A Review. Int J Cancer (2016) 138:2570-8. doi: 10.1002/ijc.29923

35. Lombardo Y, de Giorgio A, Coombes CR, Stebbing J, Castellano L. Mammosphere Formation Assay From Human Breast Cancer Tissues and Cell Lines. J Vis Exp JoVE (2015) 97:52671. doi: 10.3791/52671

36. Manuel Iglesias J, Beloqui I, Garcia-Garcia F, Leis O, Vazquez-Martin A, Eguiara A, et al. Mammosphere Formation in Breast Carcinoma Cell Lines Depends Upon Expression of E-Cadherin. PloS One (2013) 8:e77281. doi: 10.1371/journal.pone.0077281

37. Moussawi FA, Kumar A, Pasquereau S, Tripathy MK, Karam W, Diab-Assaf M, et al. The Transcriptome of Human Mammary Epithelial Cells Infected With the HCMV-DB Strain Displays Oncogenic Traits. Sci Rep (2018) 8:12574. doi: 10.1038/s41598-018-30109-1

38. Klopp AH, Lacerda L, Gupta A, Debeb BG, Solley T, Li L, et al. Mesenchymal Stem Cells Promote Mammosphere Formation and Decrease E-Cadherin in Normal and Malignant Breast Cells. PloS One (2010) 5:e12180. doi: 10.1371/ journal.pone. 0012180

39. Renzette N, Gibson L, Bhattacharjee B, Fisher D, Schleiss MR, Jensen JD, et al. Rapid Intrahost Evolution of Human Cytomegalovirus Is Shaped by Demography and Positive Selection. PloS Genet (2013) 9:e1003735. doi: 10.1371/journal.pgen.1003735

40. Chan G, Nogalski MT, Yurochko AD. Activation of EGFR on Monocytes is Required for Human Cytomegalovirus Entry and Mediates Cellular Motility. Proc Natl Acad Sci USA (2009) 106:22369-74. doi: 10.1073/pnas. 0908787106

41. Grivennikov SI, Greten FR, Karin M. Immunity, Inflammation, and Cancer. Cell (2010) 140:883-99. doi: 10.1016/j.cell.2010.01.025

42. Teng MW, Bolovan-Fritts C, Dar RD, Womack A, Simpson ML, Shenk T, et al. An Endogenous Accelerator for Viral Gene Expression Confers a Fitness Advantage. Cell (2012) 151:1569-80. doi: 10.1016/j.cell.2012.11.051

43. McKinney C, Zavadil J, Bianco C, Shiflett L, Brown S, Mohr I. Global Reprogramming of the Cellular Translational Landscape Facilitates Cytomegalovirus Replication. Cell Rep (2014) 6:9-17. doi: 10.1016/ j.celrep.2013.11.045

44. Dimri G, Band H, Band V. Mammary Epithelial Cell Transformation: Insights From Cell Culture and Mouse Models. Breast Cancer Res BCR (2005) 7:171-9. doi: $10.1186 /$ bcr 1275

45. Abba MC, Laguens RM, Dulout FN, Golijow CD. The C-Myc Activation in Cervical Carcinomas and HPV 16 Infections. Mutat Res (2004) 557:151-8. doi: 10.1016/j.mrgentox.2003.10.005

46. Lee EYHP, Muller WJ. Oncogenes and Tumor Suppressor Genes. Cold Spring Harb Perspect Biol (2010) 2:a003236. doi: 10.1101/cshperspect.a003236 
47. Hannemann H, Rosenke K, O’Dowd JM, Fortunato EA. The Presence of P53 Influences the Expression of Multiple Human Cytomegalovirus Genes at Early Times Postinfection. J Virol (2009) 83:4316-25. doi: 10.1128/JVI.02075-08

48. Muganda P, Mendoza O, Hernandez J, Qian Q. Human Cytomegalovirus Elevates Levels of the Cellular Protein P53 in Infected Fibroblasts. J Virol (1994) 68:8028-34. doi: 10.1128/JVI.68.12.8028-8034.1994

49. Hsu C-H, Chang MDT, Tai K-Y, Yang Y-T, Wang P-S, Chen C-J, et al. HCMV IE2-Mediated Inhibition of HAT Activity Downregulates P53 Function. EMBO J (2004) 23:2269-80. doi: 10.1038/sj.emboj.7600239

50. Helt A-M, Galloway DA. Mechanisms by Which DNA Tumor Virus Oncoproteins Target the $\mathrm{Rb}$ Family of Pocket Proteins. Carcinogenesis (2003) 24:159-69. doi: 10.1093/carcin/24.2.159

51. Cress WD, Nevins JR. Use of the E2F Transcription Factor by DNA Tumor Virus Regulatory Proteins. In: Farnham PJ, editor. Transcriptional Control of Cell Growth: The E2F Gene Family Current Topics in Microbiology and Immunology. Berlin, Heidelberg: Springer (1996). p. 63-78. doi: 10.1007/ 978-3-642-79910-5_3

52. Elenbaas B, Spirio L, Koerner F, Fleming MD, Zimonjic DB, Donaher JL, et al. Human Breast Cancer Cells Generated by Oncogenic Transformation of Primary Mammary Epithelial Cells. Genes Dev (2001) 15:50-65. doi: 10.1101/gad.828901

53. Soroceanu L, Cobbs CS. Is HCMV a Tumor Promoter? Virus Res (2011) 157:193-203. doi: 10.1016/j.virusres.2010.10.026

54. Kalejta RF, Bechtel JT, Shenk T. Human Cytomegalovirus Pp71 Stimulates Cell Cycle Progression by Inducing the Proteasome-Dependent Degradation of the Retinoblastoma Family of Tumor Suppressors. Mol Cell Biol (2003) 23:1885-95. doi: 10.1128/MCB.23.6.1885-1895.2003

55. Kalejta RF, Shenk T. Proteasome-Dependent, Ubiquitin-Independent Degradation of the Rb Family of Tumor Suppressors by the Human Cytomegalovirus Pp71 Protein. Proc Natl Acad Sci USA (2003) 100:3263-8. doi: 10.1073/pnas.0538058100

56. Hume AJ, Finkel JS, Kamil JP, Coen DM, Culbertson MR, Kalejta RF. Phosphorylation of Retinoblastoma Protein by Viral Protein With Cyclin-Dependent Kinase Function. Science (2008) 320:797-9. doi: 10.1126/science.1152095

57. Poma EE, Kowalik TF, Zhu L, Sinclair JH, Huang ES. The Human Cytomegalovirus IE1-72 Protein Interacts With the Cellular P107 Protein and Relieves P107-Mediated Transcriptional Repression of an E2F-Responsive Promoter. J Virol (1996) 70:7867-77. doi: 10.1128/JVI.70.11.7867-7877.1996

58. Wiebusch L, Asmar J, Uecker R, Hagemeier C. Human Cytomegalovirus Immediate-Early Protein 2 (IE2)-Mediated Activation of Cyclin E Is CellCycle-Independent and Forces S-Phase Entry in IE2-Arrested Cells. J Gen Virol (2003) 84:51-60. doi: 10.1099/vir.0.18702-0

59. Song Y-J, Stinski MF. Effect of the Human Cytomegalovirus IE86 Protein on Expression of E2F-Responsive Genes: A DNA Microarray Analysis. Proc Natl Acad Sci USA (2002) 99:2836-41. doi: 10.1073/pnas.052010099

60. Boldogh I, AbuBakar S, Deng CZ, Albrecht T. Transcriptional Activation of Cellular Oncogenes Fos, Jun, and Myc by Human Cytomegalovirus. J Virol (1991) 65:1568-71. doi: 10.1128/JVI.65.3.1568-1571.1991

61. Boldogh I, AbuBakar S, Albrecht T. Activation of Proto-Oncogenes: An Immediate Early Event in Human Cytomegalovirus Infection. Science (1990) 247:561-4. doi: 10.1126/science.1689075

62. Hagemeier C, Walker SM, Sissons PJ, Sinclair JH. The 72k IE1 and 80K IE2 Proteins of Human Cytomegalovirus Independently Trans-Activate the CFos, C-Myc and Hsp70 Promoters via Basal Promoter Elements. J Gen Virol (1992) 73(Pt 9):2385-93. doi: 10.1099/0022-1317-73-9-2385

63. Altomare DA, Testa JR. Perturbations of the AKT Signaling Pathway in Human Cancer. Oncogene (2005) 24:7455-64. doi: 10.1038/sj.onc.1209085

64. Zhao JJ, Gjoerup OV, Subramanian RR, Cheng Y, Chen W, Roberts TM, et al. Human Mammary Epithelial Cell Transformation Through the Activation of Phosphatidylinositol 3-Kinase. Cancer Cell (2003) 3:483-95. doi: 10.1016/ s1535-6108(03)00088-6

65. Cojohari O, Peppenelli MA, Chan GC. Human Cytomegalovirus Induces an Atypical Activation of Akt To Stimulate the Survival of Short-Lived Monocytes. J Virol (2016) 90:6443-52. doi: 10.1128/JVI.00214-16

66. Gilmore TD, Koedood M, Piffat KA, White DW. Rel/NF-Kappab/IkappaB Proteins and Cancer. Oncogene (1996) 13:1367-78.

67. Luque I, Gélinas C. Rel/NF-Kappa B and I Kappa B Factors in Oncogenesis. Semin Cancer Biol (1997) 8:103-11. doi: 10.1006/scbi.1997.0061
68. Luo J, Yan R, He X, He J. Constitutive Activation of STAT3 and Cyclin D1 Overexpression Contribute to Proliferation, Migration and Invasion in Gastric Cancer Cells. Am J Transl Res (2017) 9:5671-7.

69. Lilley CE, Carson CT, Muotri AR, Gage FH, Weitzman MD. DNA Repair Proteins Affect the Lifecycle of Herpes Simplex Virus 1. Proc Natl Acad Sci USA (2005) 102:5844-9. doi: 10.1073/pnas.0501916102

70. Tarakanova Vl, Leung-Pineda V, Hwang S, Yang Cw, Matatall K, Basson M, et al. Gamma-Herpesvirus Kinase Actively Initiates a DNA Damage Response by Inducing Phosphorylation of H2AX to Foster Viral Replication. Cell Host Microbe (2007) 1:275-86. doi: 10.1016/j.chom. 2007.05.008

71. Moody CA, Laimins LA. Human Papillomaviruses Activate the ATM DNA Damage Pathway for Viral Genome Amplification Upon Differentiation. PloS Pathog (2009) 5:e1000605. doi: 10.1371/journal.ppat.1000605

72. Xiaofei E, Pickering MT, Debatis M, Castillo J, Lagadinos A, Wang S, et al. An E2F1-Mediated DNA Damage Response Contributes to the Replication of Human Cytomegalovirus. PloS Pathog (2011) 7:e1001342. doi: 10.1371/ journal.ppat.1001342

73. Perfettini J-L, Nardacci R, Bourouba M, Subra F, Gros L, Séror C, et al. Critical Involvement of the ATM-Dependent DNA Damage Response in the Apoptotic Demise of HIV-1-Elicited Syncytia. PloS One (2008) 3:e2458. doi: 10.1371/journal.pone. 0002458

74. Castillo JP, Frame FM, Rogoff HA, Pickering MT, Yurochko AD, Kowalik TF. Human Cytomegalovirus IE1-72 Activates Ataxia Telangiectasia Mutated Kinase and a P53/P21-Mediated Growth Arrest Response. J Virol (2005) 79:11467-75. doi: 10.1128/JVI.79.17.11467-11475.2005

75. Xiaofei E, Savidis G, Chin CR, Wang S, Lu S, Brass AL, et al. A Novel DDB2ATM Feedback Loop Regulates Human Cytomegalovirus Replication. J Virol (2014) 88:2279-90. doi: 10.1128/JVI.03423-13

76. Shirata N, Kudoh A, Daikoku T, Tatsumi Y, Fujita M, Kiyono T, et al. Activation of Ataxia Telangiectasia-Mutated DNA Damage Checkpoint Signal Transduction Elicited by Herpes Simplex Virus Infection. J Biol Chem (2005) 280:30336-41. doi: 10.1074/jbc.M500976200

77. Mohni KN, Mastrocola AS, Bai P, Weller SK, Heinen CD. DNA Mismatch Repair Proteins Are Required for Efficient Herpes Simplex Virus 1 Replication $\nabla$. J Virol (2011) 85:12241-53. doi: 10.1128/JVI.05487-11

78. Chakraborty U, Dinh TA, Alani E. Genomic Instability Promoted by Overexpression of Mismatch Repair Factors in Yeast: A Model for Understanding Cancer Progression. Genetics (2018) 209:439-56. doi: 10.1534/genetics.118.300923

79. Wilczak W, Rashed S, Hube-Magg C, Kluth M, Simon R, Büscheck F, et al. Up-Regulation of Mismatch Repair Genes MSH6, PMS2 and MLH1 Parallels Development of Genetic Instability and is Linked to Tumor Aggressiveness and Early PSA Recurrence in Prostate Cancer. Carcinogenesis (2017) 38:1927. doi: $10.1093 /$ carcin/bgw116

80. Yousef EM, Tahir MR, St-Pierre Y, Gaboury LA. MMP-9 Expression Varies According to Molecular Subtypes of Breast Cancer. BMC Cancer (2014) 14:609. doi: 10.1186/1471-2407-14-609

81. Ballin M, Gomez DE, Sinha CC, Thorgeirsson UP. Ras Oncogene Mediated Induction of a 92kda Metalloproteinase; Strong Correlation With the Malignant Phenotype. Biochem Biophys Res Commun (1988) 154:832-8. doi: 10.1016/0006-291X(88)90215-X

82. Bernhard EJ, Gruber SB, Muschel RJ. Direct Evidence Linking Expression of Matrix Metalloproteinase 9 (92-kDa Gelatinase/Collagenase) to the Metastatic Phenotype in Transformed Rat Embryo Cells. Proc Natl Acad Sci (1994) 91:4293-7. doi: 10.1073/pnas.91.10.4293

83. Björklund M, Koivunen E. Gelatinase-Mediated Migration and Invasion of Cancer Cells. Biochim Biophys Acta (2005) 1755:37-69. doi: 10.1016/ j.bbcan.2005.03.001

84. Shay G, Lynch CC, Fingleton B. Moving Targets: Emerging Roles for MMPs in Cancer Progression and Metastasis. Matrix Biol J Int Soc Matrix Biol (2015) 44-46:200-6. doi: 10.1016/j.matbio.2015.01.019

85. Diaz N, Minton S, Cox C, Bowman T, Gritsko T, Garcia R, et al. Activation of Stat3 in Primary Tumors From High-Risk Breast Cancer Patients Is Associated With Elevated Levels of Activated Src and Survivin Expression. Clin Cancer Res (2006) 12:20-8. doi: 10.1158/1078-0432.CCR-04-1749

86. Xu J, Lamouille S, Derynck R. TGF- $\beta$-Induced Epithelial to Mesenchymal Transition. Cell Res (2009) 19:156-72. doi: 10.1038/cr.2009.5 
87. Wendt MK, Allington TM, Schiemann WP. Mechanisms of EpithelialMesenchymal Transition by TGF- $\beta$. Future Oncol Lond Engl (2009) 5:114568. doi: $10.2217 /$ fon. 09.90

88. Mrouj K, Andrés-Sánchez N, Dubra G, Singh P, Sobecki M, Chahar D, et al. Ki-67 Regulates Global Gene Expression and Promotes Sequential Stages of Carcinogenesis. Proc Natl Acad Sci (2021) 118:e2026507118. doi: 10.1073/ pnas. 2026507118

89. Li LT, Jiang G, Chen Q, Zheng JN. Ki67 is a Promising Molecular Target in the Diagnosis of Cancer (Review). Mol Med Rep (2015) 11:1566-72. doi: 10.3892/ mmr.2014.2914

90. Keam B, Im S-A, Lee K-H, Han S-W, Oh D-Y, Kim JH, et al. Ki-67 can be Used for Further Classification of Triple Negative Breast Cancer Into Two Subtypes With Different Response and Prognosis. Breast Cancer Res (2011) 13:R22. doi: $10.1186 / \mathrm{bcr} 2834$

91. Li H, Han X, Liu Y, Liu G, Dong G. Ki67 as a Predictor of Poor Prognosis in Patients With Triple-Negative Breast Cancer. Oncol Lett (2015) 9:149-52. doi: $10.3892 / \mathrm{ol} .2014 .2618$
Conflict of Interest: The authors declare that the research was conducted in the absence of any commercial or financial relationships that could be construed as a potential conflict of interest.

Publisher's Note: All claims expressed in this article are solely those of the authors and do not necessarily represent those of their affiliated organizations, or those of the publisher, the editors and the reviewers. Any product that may be evaluated in this article, or claim that may be made by its manufacturer, is not guaranteed or endorsed by the publisher.

Copyright (๑ 2021 Haidar Ahmad, Pasquereau, El Baba, Nehme, Lewandowski and Herbein. This is an open-access article distributed under the terms of the Creative Commons Attribution License (CC BY). The use, distribution or reproduction in other forums is permitted, provided the original author(s) and the copyright owner(s) are credited and that the original publication in this journal is cited, in accordance with accepted academic practice. No use, distribution or reproduction is permitted which does not comply with these terms. 